\title{
Pyrolysis of azetidinone derivatives: a versatile route towards electron-rich alkenes, C-1 allylation and/or homologation of aldehydes $\dagger$
}

\author{
Nouf S. Al-Hamdan, Osama M. Habib, Yehia A. Ibrahim, Nouria A. Al-Awadi* \\ and Osman M. E. El-Dusouqui
}

Pyrolysis of $\beta$-lactams and $\beta$-thiolactams led essentially to stereoselective synthesis of the high energy electron-rich Z-alkenes. Extension of this methodology to the pyrolysis of 3 -allyloxy derivatives gave a simple direct route to the synthetically important 4-pentenal. These pyrolytic transformations convert aldehydes to aryloxyalkenes (a protected homologation) and 4-pentenal (a C-1 allylation and homologation). The starting 3-aryloxy and 3-allyloxy- $\beta$-lactams were synthesized by the standard Staudinger ketene-imine $[2+2]$ cycloaddition. The corresponding $\beta$-thiolactams have readily been obtained in good yields by thiation of $\beta$-lactams with Lawesson's reagent.

Received 5th February 2014 Accepted 22nd April 2014

DOI: $10.1039 / \mathrm{c} 4 \mathrm{ra01024h}$

www.rsc.org/advances reagents for further chemical elaboration. Scheme 2 illustrates our strategy to utilize such pyrolytic transformation to convert aldehydes to aryloxyalkenes (a protected homologation of the starting aldehyde) and 4-pentenal (a C-1 allylation and homologation).

997, ${ }^{1}$ since then azetidin-2-ones have acquired a prominent place in organic chemistry as synthons for further elaboration. The constrained azetidinone ring has been employed successfully in synthetic methodologies towards all kinds of nitrogencontaining compounds. ${ }^{2}$ The Staudinger $[2+2]$ ketene-imine cycloaddition reaction is considered as one of the most important synthetic approaches to $\beta$-lactams (2-azetidinones) which have important application in pharmaceutical and synthetic chemistry. ${ }^{\mathbf{1 - 5}}$ Although the reaction has been discovered a hundred years ago, ${ }^{4}$ it still attracts recent interest. ${ }^{1-3,5}$

Pyrolysis of these $\beta$-lactams has received little attention and was shown to give mainly alkenes with no more functional groups. ${ }^{6-12}$ The thermal fragmentation of $\beta$-lactams occurs by two different pathways, the first one gives the starting ketene and imine (B) and the second pathway (A) gives alkene and isocyanate which could be trapped with amine to give the corresponding urea derivatives, Fischer found that the thermal fragmentation favours pathway A, while photolysis favours pathway B (Scheme 1). ${ }^{\mathbf{1 1}}$

The present work is part of a project directed towards exploring the pyrolytic behaviour of this important ring system and its potentiality in producing useful functionalized organic

Chemistry Department, Faculty of science, Kuwait University, P.O. Box 5969, Safat 13060, Kuwait. E-mail: n.alawadi@ku.edu.kw; Fax: +96524816482; Tel: +96524985537

$\dagger$ Electronic supplementary information (ESI) available: Characterization data for all new $\beta$-lactams and $\beta$-thiolactams including ${ }^{1} \mathrm{H}$ NMR and ${ }^{13} \mathrm{C}$ NMR spectra (Tables 1 and 2) and mass spectra of some representative examples. See DOI: 10.1039/c4ra01024h

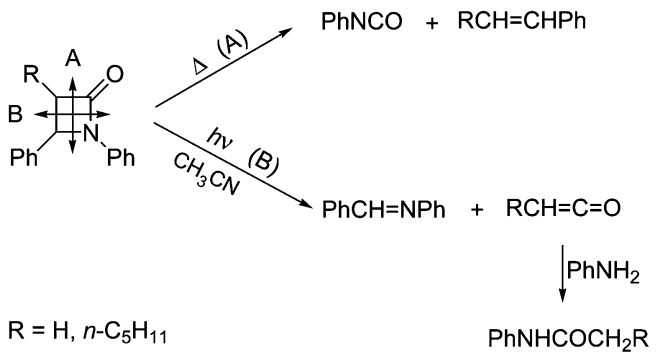

Scheme 1 Routes for fragmentation of $\beta$-lactams.

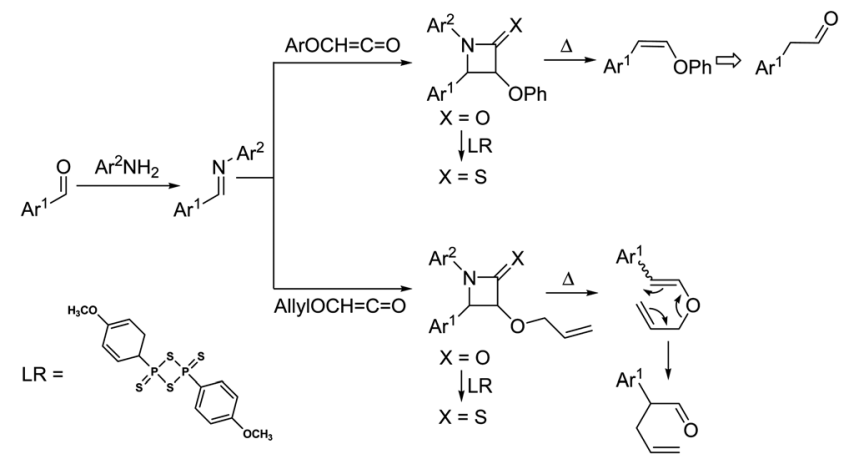

Scheme 2 Strategy to convert aldehydes to aryloxyalkenes and 4pentenal through $\beta$-lactams and thio- $\beta$-lactams pyrolysis. 


\section{Results and discussion}

The starting $\beta$-lactams and their corresponding 2 -thioxo derivatives needed in our study were synthesized as outlined in Scheme 3. Thus, the reaction of imines $1 \mathbf{a}-\mathbf{g}$ with excess phenoxyacetyl chloride (2.5 equiv.) in dry DCM $\left(\mathrm{CH}_{2} \mathrm{Cl}_{2}\right)$ in the presence of triethylamine with exception of $1 \mathbf{d}$ gave the corresponding $\beta$-lactams as cis isomer $\mathbf{2 a - c , e - g}$ respectively. In case of $\mathbf{1 d}$ a mixture of cis and trans $\beta$-lactams $\mathbf{2 d}$ and $\mathbf{3 d}$ were formed (40:60) respectively. The cis stereochemistry of all products $2 \mathbf{a}-$ g was assigned based on the coupling constants between the protons at C3 and C4, with observed $J$-values $=4.8 \mathrm{~Hz} .^{13}$ The trans stereoisomer 3d was unambiguously assigned based on the coupling constant between the protons at $\mathrm{C} 3$ and $\mathrm{C} 4$, with the observed $J$-value $=1.8 \mathrm{~Hz}$ (ref. 13) (see ESI Table $1 \dagger$ ).

Moreover, it seemed worthwhile to compare the pyrolytic reaction of $\beta$-lactams $\mathbf{2} \mathbf{a}-\mathbf{g}$ and the corresponding thio- $\beta$-lactams 4a-g. The later were synthesized in $65-73 \%$ yields by refluxing the corresponding $\beta$-lactams $\mathbf{2 a - g}$ with Lawesson's reagent (LR) in dry toluene for $3 \mathrm{~h}$ (Scheme 3). All products $\mathbf{4 a - g}$ retained the cis stereochemistry as indicated by the coupling constants between the protons at $\mathrm{C} 3$ and $\mathrm{C} 4$, with the observed $J$-values $=4.4-4.8 \mathrm{~Hz}$.

We first studied the static pyrolysis (sealed-tube pyrolysis) of 2a to optimize the condition for complete reaction for the desired pyrolysis products. The products of the reaction were analyzed directly after the thermolysis using ${ }^{1} \mathrm{H}$ and ${ }^{13} \mathrm{C}$ NMR spectroscopy. Table 1 shows the main products obtained under reduced pressure $1 \times 10^{-2}$ Torr and at different pyrolysis temperatures $\left(250,270,290,300,320,350\right.$ and $\left.400{ }^{\circ} \mathrm{C}\right)$. It is clear

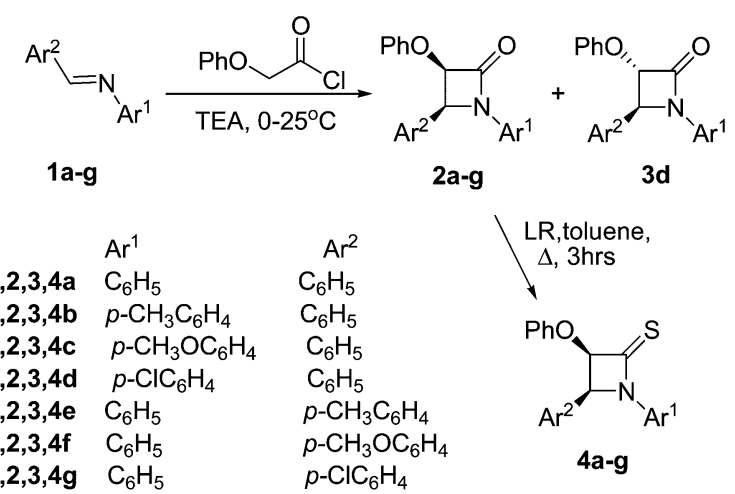

Scheme 3 Synthesis of $\beta$-lactams $2 \mathrm{a}-\mathrm{g}$ and thio- $\beta$-lactams $4 \mathrm{a}-\mathrm{g}$ that the substrate 2a is stable up to $250{ }^{\circ} \mathrm{C}$ and start to pyrolyze at $270{ }^{\circ} \mathrm{C}$ to $25 \%$ of the corresponding $Z$-alkene. However, at temperature lower $300{ }^{\circ} \mathrm{C}$ isomerisation of cis- $\beta$-lactam $2 \mathrm{a}$ to trans- $\beta$-lactam 3a was the main process, while by increasing the temperature and thermolysis time, fission of the $\beta$-lactam ring became the major pathway. Complete pyrolysis of 2a occurred at $350{ }^{\circ} \mathrm{C}$ in 20 minutes with the formation of the $Z$ and $E$ alkene in $37 \%$ yield. Increasing the pyrolysis temperature to $400{ }^{\circ} \mathrm{C}$ and reducing the reaction time to 5 minutes increased $Z: E$ ratio (Table 1).

The $\beta$-lactams $2 \mathrm{a}-\mathrm{g}$ subjected to static pyrolysis at $350{ }^{\circ} \mathrm{C}$ and flash vacuum pyrolysis (FVP) at $600{ }^{\circ} \mathrm{C}$. The pyrolytic reaction mixtures was carefully analyzed with the use of ${ }^{1} \mathrm{H}$ and ${ }^{13} \mathrm{C}$ NMR spectra, we found that, in STP at $350{ }^{\circ} \mathrm{C}$ of $\beta$-lactams $2 \mathbf{a}-\mathbf{g}$ in addition to formation of the corresponding $E$ and $Z$ alkenes, imines $\mathbf{1 a}-\mathbf{g}$ were formed in $\mathbf{6}-\mathbf{1 1} \%$ yield. The main cleavage reaction is postulated to involve a retro $[2+2]$ cycloreversion type fragmentation of the $\beta$-lactam ring liberating $Z$-alkene isomers together with the $E$ isomers in a high diastereomeric ratio, however, under FVT the $Z$ isomer is the major products in all cases (Scheme 4, Table 2). This could be explained by the fact the long residence time in static pyrolysis leads to isomerization of the $Z$ isomer to the more stable $E$ isomer.

Similar study revealed that the optimum temperature for the static pyrolysis of the thiolactams $4 \mathrm{a}-\mathrm{g}$ is $280{ }^{\circ} \mathrm{C}$ and $550{ }^{\circ} \mathrm{C}$ for the FVP. At $250^{\circ} \mathrm{C}$ (STP) only partial isomerisation took place to give the corresponding trans-thio- $\beta$-lactams 6. Thus STP (at 250 ${ }^{\circ} \mathrm{C}, 20 \mathrm{~min}$ ) of $\mathbf{4 a}, \mathbf{c}, \mathbf{f}$ gave a mixture of $\mathbf{4 a}, \mathbf{c}, \mathbf{f}$ with the corresponding trans isomers 6a (25\%), 6c (37\%), 6f (40\%).

Moreover, the corresponding thiourea derivatives 13a-d (11$16 \%$ ) instead of arylisocyanates were detected in all cases. Unfortunately at higher temperature $280{ }^{\circ} \mathrm{C}$ for $15 \mathrm{~min}$ charring took place with formation of poor yield of the corresponding $E$ and $Z$ alkenes (5-14\%). On the other hand, FVP of thio- $\beta$-lactams $4 \mathrm{a}-\mathrm{g}$ at $550{ }^{\circ} \mathrm{C}$ gave better yield of the corresponding alkenes (Scheme 4, Table 2) with higher $Z$ diastereomeric ratio.

The $5 E / Z$ isomers percent were calculated depending on the integration of the $E$ isomer proton $\left(\mathrm{Ar}^{2}-\mathrm{CH}=\right)$ at $\delta=6.3 \mathrm{ppm}(J$ $=12.8 \mathrm{~Hz})$ with the integration of the $Z$ isomer proton $\left(\mathrm{Ar}^{2}-\right.$ $\mathrm{CH}=)$ at $\delta=5.6 \mathrm{ppm}(J=6.8 \mathrm{~Hz})$, and all products percent are calculated using DCM method. ${ }^{14}$

Our study was then extended to pyrolysis of the 3-allyloxy analogues of compounds $\mathbf{2}$ and $\mathbf{4}$ which are expected to undergo further Claisen rearrangement for the initially pyrolytically produced allyloxyalkene. The new 3-allyloxy-1,4-diaryl-2-

Table 1 Products from STP of cis- $\beta$-lactam $2 a$ (yield \%)

\begin{tabular}{|c|c|c|c|c|c|c|}
\hline Entry & Temp. $\left({ }^{\circ} \mathrm{C}\right)$ & Time (min) & cis- $\beta$-lactam 2a & trans- $\beta$-lactam 3a & $Z-\mathbf{5 a}$ & $E-\mathbf{5 a}$ \\
\hline 1 & 250 & 30 & 100 & 0 & 0 & 0 \\
\hline 2 & 270 & 90 & 40 & 25 & 25 & 0 \\
\hline 3 & 290 & 15 & 50 & 20 & 15 & 0 \\
\hline 4 & 300 & 30 & 25 & 25 & 25 & 1 \\
\hline 5 & 320 & 30 & 8 & 18 & 23 & 13 \\
\hline 6 & 350 & 20 & 0 & 0 & 20 & 17 \\
\hline 7 & 400 & 5 & 0 & 0 & 21 & 10 \\
\hline
\end{tabular}



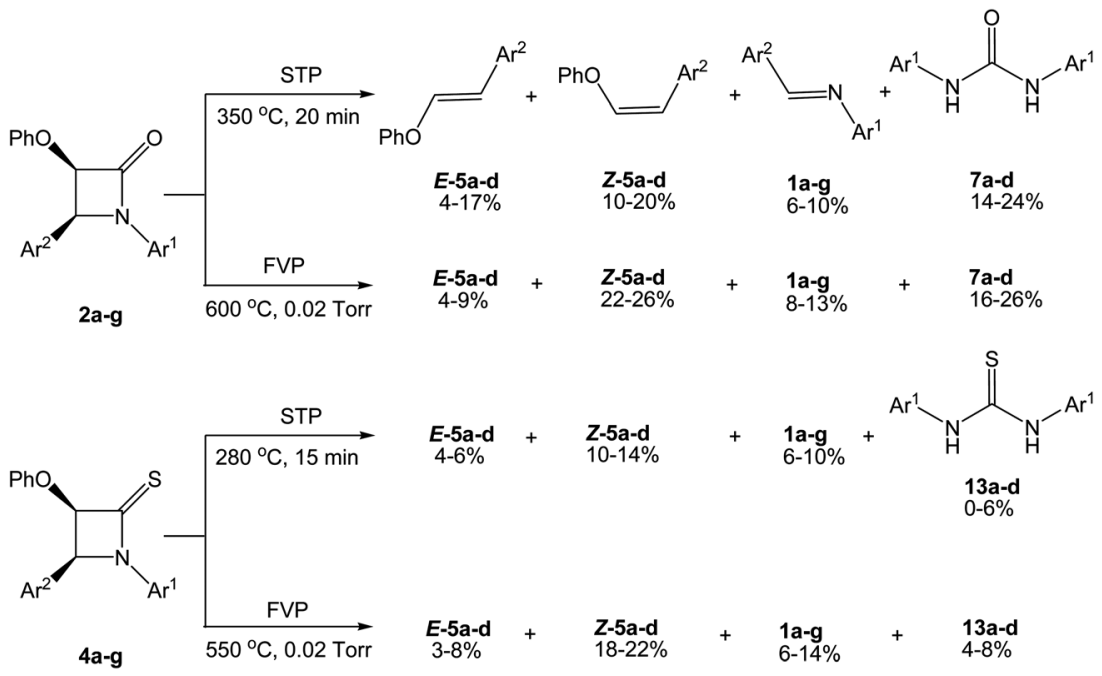

$\begin{array}{lll} & \mathrm{Ar}^{1} & \mathrm{Ar}^{2} \\ \mathbf{1 - 7 , 1 0 , 1 3 a} & \mathrm{C}_{6} \mathrm{H}_{5} & \mathrm{C}_{6} \mathrm{H}_{5} \\ \mathbf{1 - 7 , 1 0 , 1 3 b} & p-\mathrm{CH}_{3} \mathrm{C}_{6} \mathrm{H}_{4} & \mathrm{C}_{6} \mathrm{H}_{5} \\ \mathbf{1 - 7 , 1 0 , 1 3 c} & p-\mathrm{CH}_{3} \mathrm{OC}_{6} \mathrm{H}_{4} & \mathrm{C}_{6} \mathrm{H}_{5} \\ \mathbf{1 - 7 , 1 0 , 1 3 d} & p-\mathrm{ClC}_{6} \mathrm{H}_{4} & \mathrm{C}_{6} \mathrm{H}_{5} \\ \mathbf{1 - 7 , 1 0 , 1 3 e} & \mathrm{C}_{6} \mathrm{H}_{5} & p-\mathrm{CH}_{3} \mathrm{C}_{6} \mathrm{H}_{4} \\ \mathbf{1 - 7 , 1 0 , 1 3 f} & \mathrm{C}_{6} \mathrm{H}_{5} & p-\mathrm{CH}_{3} \mathrm{OC}_{6} \mathrm{H}_{4} \\ \mathbf{1 - 7 , 1 0 , 1 3 g} & \mathrm{C}_{6} \mathrm{H}_{5} & p-\mathrm{ClC}_{6} \mathrm{H}_{4}\end{array}$

Scheme 4 Products of SVP and FVP of cis $\beta$-lactam $2 \mathrm{a}-\mathrm{g}$ and cis-thio- $\beta$-lactams $4 \mathrm{a}-\mathrm{g}$.

Table 2 Products of STP and FVP of cis- $\beta$-lactams $2 \mathrm{a}-\mathrm{g}$ and cis-thio- $\beta$-lactams $4 \mathrm{a}-\mathrm{g}$

\begin{tabular}{|c|c|c|c|c|c|c|}
\hline \multirow[b]{2}{*}{ Comp. no. } & \multicolumn{3}{|l|}{$\underline{\mathrm{STP}^{a}}$} & \multicolumn{3}{|l|}{$\mathrm{FVP}^{b}$} \\
\hline & $\begin{array}{l}\text { Alkene } \\
(E / Z)\end{array}$ & $\begin{array}{l}\text { Imine } \\
\text { (yield \%) }\end{array}$ & $\begin{array}{l}\text { Urea (thiourea) } \\
\text { (yield \%) }\end{array}$ & $\begin{array}{l}\text { Alkene } \\
(E / Z)\end{array}$ & $\begin{array}{l}\text { Imine } \\
\text { (yield \%) }\end{array}$ & $\begin{array}{l}\text { Urea (thiourea) } \\
\text { (yield \%) }\end{array}$ \\
\hline $2 a$ & $5 a(17 / 20)$ & $1 \mathbf{a}(10)$ & $7 \mathbf{a}(24)$ & $5 \mathbf{a}(6 / 25)$ & 1a (11) & $7 \mathbf{a}(18)$ \\
\hline $2 \mathbf{b}$ & $5 a(11 / 16)$ & $\mathbf{1 b}(8)$ & $7 \mathbf{b}(20)$ & $5 \mathbf{a}(9 / 22)$ & $1 \mathbf{b}(10)$ & $7 \mathbf{b}(16)$ \\
\hline $2 \mathrm{c}$ & $5 \mathbf{a}(9 / 17)$ & $1 c(7)$ & $7 c(20)$ & $5 a(6 / 23)$ & $1 c(12)$ & $7 c(26)$ \\
\hline $2 f$ & $5 c(9 / 12)$ & $\mathbf{1 f}(6)$ & $7 a(14)$ & $5 c(4 / 25)$ & 1f $(13)$ & $7 a(20)$ \\
\hline $2 \mathrm{~g}$ & $5 d(16 / 22)$ & $1 \mathrm{~g}(11)$ & $7 a(24)$ & $5 d(7 / 24)$ & $1 \mathrm{~g}(12)$ & $7 a(24)$ \\
\hline $4 a$ & $5 \mathbf{a}(6 / 10)$ & $1 \mathrm{a}(8)$ & $13 a(4)$ & $5 \mathbf{a}(4 / 18)$ & 1a $(7)$ & $13 a(8)$ \\
\hline $4 b$ & $5 \mathbf{a}(5 / 10)$ & $1 b(10)$ & $13 \mathbf{b}(0)$ & $5 \mathbf{a}(7 / 20)$ & $1 \mathbf{b}(9)$ & $13 b(4)$ \\
\hline $4 c$ & $5 \mathbf{a}(6 / 14)$ & $1 c(6)$ & $13 c(4)$ & $5 \mathbf{a}(3 / 22)$ & $1 c(11)$ & $13 c(8)$ \\
\hline 4d & $5 \mathbf{a}(4 / 12)$ & 1d (8) & 13d (6) & $5 a(5 / 19)$ & 1d (6) & 13d (6) \\
\hline
\end{tabular}

${ }^{a} \mathrm{STP}, 350{ }^{\circ} \mathrm{C}, 0.01$ Torr, $20 \mathrm{~min}$. for $\beta$-lactams $2 \mathbf{a}-\mathbf{g}, 280{ }^{\circ} \mathrm{C}, 0.01$ Torr, $15 \mathrm{~min}$ for thio- $\beta$-lactams $4 \mathrm{a}-\mathrm{g} .{ }^{b} \mathrm{FVP}, 600{ }^{\circ} \mathrm{C}, 0.02$ Torr for $\beta$-lactams $2 \mathrm{a}-\mathrm{g}$, $550{ }^{\circ} \mathrm{C}, 0.02$ Torr; for thio- $\beta$-lactams $4 \mathrm{4a}-\mathrm{g}$.

azetidinone 8a-e were prepared by the reaction of imines 1a-e with allyloxyacetyl chloride in the presence of triethylamine. In all cases cis- $\beta$-lactams 8a-e were obtained except in case of the imine 1a trans- $\beta$-lactams 9a was formed in $4 \%$ yield. The corresponding 3-allyloxy- $\beta$-thiolactams 10a-e were obtained by thiation of 8a-e with Lawesson's reagent (Scheme 5) (see ESI Table $2 \dagger$ ).

The optimum temperature for pyrolysis of allyloxy-1,4-diaryl2-azetidinone 8a-e is $300{ }^{\circ} \mathrm{C}(\mathrm{STP})$ and $600{ }^{\circ} \mathrm{C}(\mathrm{FVP})$. The ${ }^{1} \mathrm{H}$ and
${ }^{13} \mathrm{C}$ NMR spectroscopic analysis of the pyrolysate showed the formation of urea derivatives $7 \mathbf{a}-\mathbf{c}$ and imines $\mathbf{1 a - c}$, but the methylene protons signal at $\delta 4.3$ corresponding to the olefinic ether intermediate $\mathbf{1 1 a}^{\mathbf{1 5 , 1 6}}$ was not detected in the pyrolysate, while the spectroscopic properties were identical with those reported for the corresponding 4-pentenal 12a-c (a Claisen rearrangement of aldehydes) $)^{17,18}$ (Scheme 6).

FVP of allyloxy-1,4-diaryl-2-azetidinone $8 \mathbf{a}-\mathbf{d}$ at $600{ }^{\circ} \mathrm{C}$ gave the 4-pentenals 12a-c together with the other by-products $7 \mathbf{a}-\mathbf{c}$, 

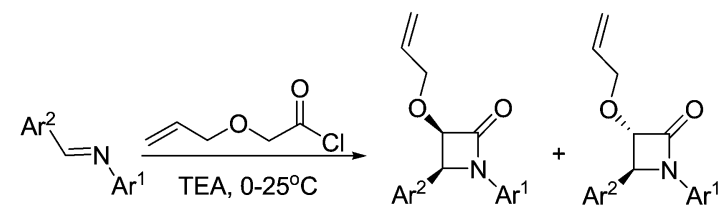

$1 a-e$

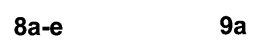

$9 a$

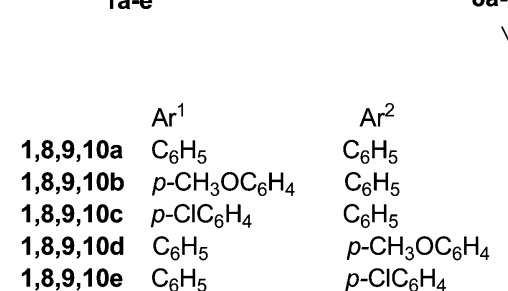

$\mathrm{LR}$, toluene
$\Delta, 3 \mathrm{hrs}$

$\checkmark$

$\begin{array}{ll}\text { 1,8,9,10a } & \mathrm{C}_{6} \mathrm{H}_{5} \\ 1,8,9,10 \text { b } & p-\mathrm{CH}_{3} \mathrm{OC}_{6} \mathrm{H}_{4} \\ 1,8,9,10 \text { c } & p-\mathrm{ClC}_{6} \mathrm{H}_{4} \\ 1,8,9,10 \text { d } & \mathrm{C}_{6} \mathrm{H}_{5} \\ 1,8,9,10 \text { e } & \mathrm{C}_{6} \mathrm{H}_{5}\end{array}$
$p-\mathrm{ClC}_{6} \mathrm{H}_{4}$

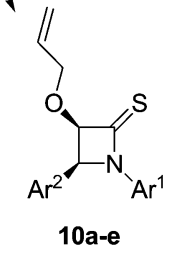

Scheme 5 Synthesis of $\beta$-lactams $8 \mathrm{a}-\mathrm{e}$ and thio- $\beta$-lactams $10 \mathrm{a}-\mathrm{e}$

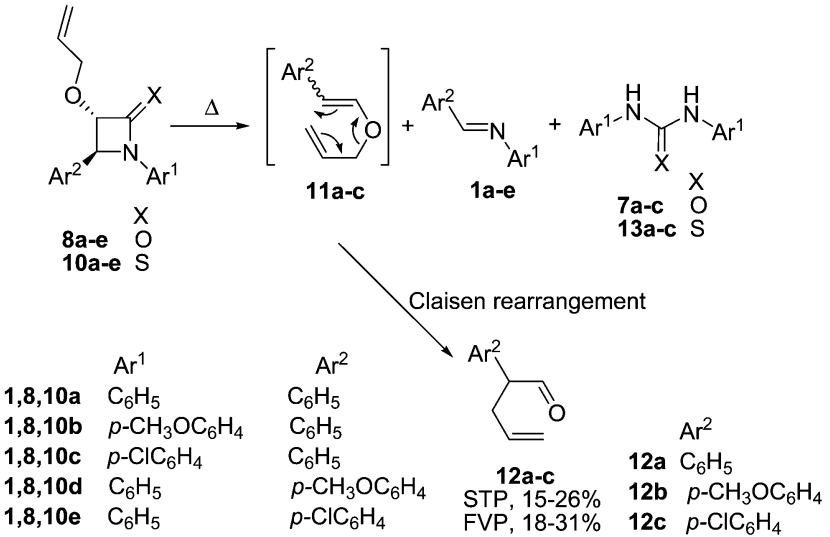

Scheme 6 Products of SVP and FVP of cis- $\beta$-lactam $8 \mathrm{a}-\mathrm{e}$ and thio- $\beta$ lactams 10a-e

1a-c but with a higher yields of aldehydes 12a-c. On the other hand, pyrolysis of thio- $\beta$-lactams 10a-e gave poor yields in both STP and FVP even on decreasing the pyrolysis temperature (270 $\left.{ }^{\circ} \mathrm{C}, \mathrm{STP}\right),\left(550{ }^{\circ} \mathrm{C}, \mathrm{FVP}\right)$. The aldehydes 12a-c and imines 1a-c have been detected in the pyrolysate in addition to traces of thiourea derivatives 13a-c (Table 3).

The percent yield of the products in pyrolysate mixture based on the characteristic protons, aldehydes based on the characteristic aldehyde proton at $9.7 \mathrm{ppm}(\mathrm{d}, J=1.6 \mathrm{~Hz}),{ }^{17,18}$ imines based on the characteristic $\mathrm{CH}=\mathrm{N}$ proton at $8.4 \mathrm{ppm}$, and urea derivatives on the exchangeable NH proton at 8.5-8.8 (ref. 19) or based on the characteristic $\mathrm{MeO}$ proton at $3.8 \mathrm{ppm}$.

\section{Conclusion}

The present work offers an interesting application of synthetic transformation of a new 2-azetidinones into valuable intermediates in synthetic chemistry including electron-rich $Z$-olefins and 4-pentenal derivatives. The latter have important applications in synthetic chemistry and were prepared recently by multi step reaction..$^{\mathbf{2 0 , 2 1}}$ Moreover, the reported synthesized aryl vinyl ethers found applications in the synthesis of biologically active molecules and natural product analogues, ${ }^{22}$ and because of its importance various methods for their synthesis have been reported. ${ }^{23}$ Also, the reported pyrolytic transformations convert aldehydes to aryloxyalkenes (a protected homologation) and 4pentenal (a C-1 allylation and homologation).

\section{Experimental}

\subsection{General}

All reactions were carried out in oven-dried glassware under $\mathrm{N}_{2}$, using commercially supplied solvents and reagents unless otherwise stated. THF and $\mathrm{CH}_{2} \mathrm{Cl}_{2}$ were redistilled from $\mathrm{Na}-$ $\mathrm{Ph}_{2} \mathrm{CO}$ and $\mathrm{CaH}_{2}$ respectively. Column chromatography was carried out on silica gel using flash techniques (eluants are given in parentheses). Analytical thin-layer chromatography was performed on precoated silica gel F254 aluminum plates with visualization under UV light.

Melting points were obtained using a melting point apparatus and are uncorrected. IR spectra were recorded on FT-IR

Table 3 Products of SVP and FVP of cis- $\beta$-lactams $8 \mathrm{a}-\mathrm{e}$ and thio- $\beta$-lactams $10 \mathrm{a}-\mathrm{e}$

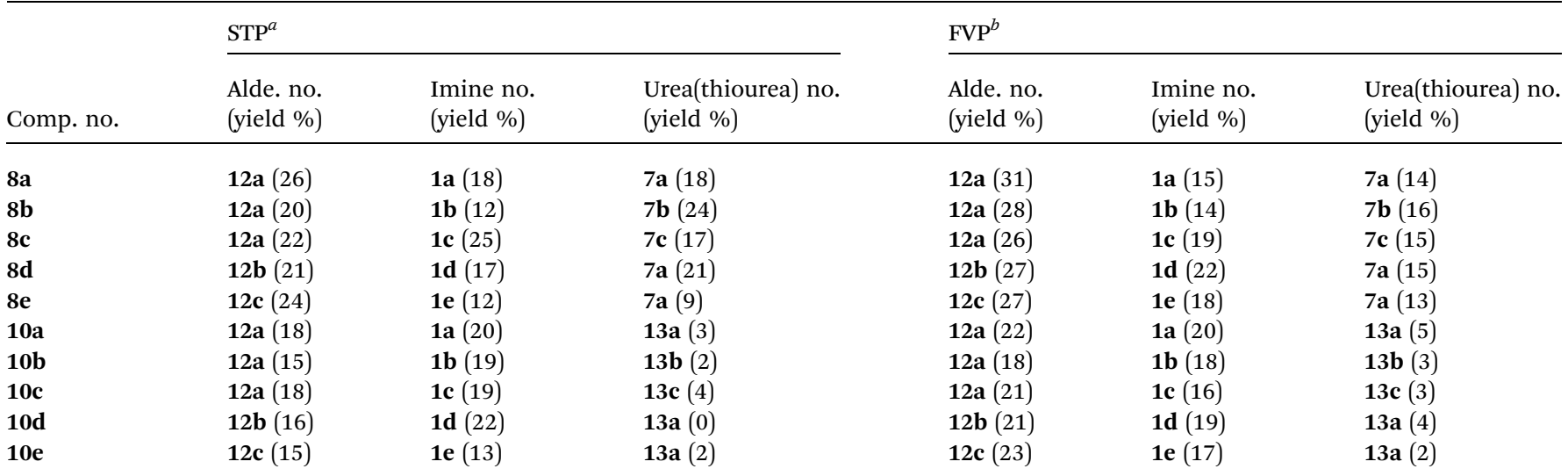

${ }^{a} \mathrm{STP}, 300{ }^{\circ} \mathrm{C}, 0.01 \mathrm{Torr}, 15 \mathrm{~min}$. for $\beta$-lactams 8a-e, $270{ }^{\circ} \mathrm{C}, 0.01$ Torr, $15 \mathrm{~min}$ for thio- $\beta$-lactams $10 \mathrm{a}-\mathbf{e} .{ }^{b} \mathrm{FVP}, 600{ }^{\circ} \mathrm{C}, 0.02 \mathrm{Torr}$ for $\beta$-lactams $8 \mathrm{a}-\mathbf{e}$, $550{ }^{\circ} \mathrm{C}, 0.02$ Torr; for thio- $\beta$-lactams 10a-e. 
spectrophotometer as neat thin films between $\mathrm{NaCl}$ plates in the case of liquid substances or as $\mathrm{KBr}$ pellets in the case of solids, with adsorptions reported in wavenumbers $\left(\mathrm{cm}^{-1}\right) .{ }^{1} \mathrm{H}$ NMR spectra were recorded at 400 or $600 \mathrm{MHz}$ with chemical shifts $(\delta)$ quoted in parts per million (ppm) and coupling constants $(J)$ recorded in hertz $(\mathrm{Hz}) .{ }^{13} \mathrm{C}$ NMR spectra were recorded at 100 or $150 \mathrm{MHz}$ with chemical shifts $(\delta)$ quoted in ppm. Mass spectral data were measured with EI positive ion mode. Microanalyses were performed on LECO CH NS-932 Elemental Analyzer.

\subsection{Synthesis of $\beta$-lactams 2a-g: general procedure}

To a stirred cold $\left(0{ }^{\circ} \mathrm{C}\right)$ solution of appropriate imines $1 \mathrm{a}-\mathrm{g}$ (40 mmol, 1 equiv.) and triethylamine ( $25 \mathrm{mmol}, 2.5$ equiv.) in dry $\mathrm{CH}_{2} \mathrm{Cl}_{2}(15 \mathrm{~mL})$ under nitrogen atmosphere was added dropwise with a syringe a solution of phenoxyacetyl chloride $(100$ mmol, 2.5 equiv.) in dry $\mathrm{CH}_{2} \mathrm{Cl}_{2}(5 \mathrm{~mL})$. The mixture was stirred at $0{ }^{\circ} \mathrm{C}$ for $30 \mathrm{~min}$, and then left stirring at room temperature overnight. The reaction mixture washed with water, $\mathrm{NaHCO}_{3}$ solution and brine. The organic layer was then dried over anhydrous $\mathrm{Na}_{2} \mathrm{SO}_{4}$ and the solvent was removed in vacuo. The remaining solid was recrystallized from ethanol to give corresponding $\beta$-lactams $\mathbf{2 a - g}$ as colourless solid in $57-74 \%$ yield.

( \pm )-cis-3-Phenoxy-1,4-diphenyl-2-azetindione (2a). Colorless crystals, yield $8.4 \mathrm{~g}$ (67\%), mp 193-195 ${ }^{\circ} \mathrm{C}\left(194{ }^{\circ} \mathrm{C}\right) ;{ }^{24} \nu_{\max }(\mathrm{KBr}) /$ $\mathrm{cm}^{-1}$ 3003, 2916, 1744, 1588, 1558, 1455, 1419, 1210, 749, 701. $\delta_{\mathrm{H}}\left(400 \mathrm{MHz}, \mathrm{CDCl}_{3}\right) 7.38-7.26(\mathrm{~m}, 9 \mathrm{H}), 7.16(\mathrm{t}, 2 \mathrm{H}, J 8.4), 7.09(\mathrm{t}$, $1 \mathrm{H}, J 7.2), 6.93$ (t, 1H, J 8.0), 6.78 (d, 2H, J 8.0), 5.57 (d, 1H, J 4.8), 5.40 (d, $1 \mathrm{H}, J 4.8) ; \delta_{\mathrm{C}}\left(100 \mathrm{MHz}, \mathrm{CDCl}_{3}\right)$ 163.3, 157.1, 137.1, 132.7, 129.49, 129.40, 128.9, 128.6, 128.3, 124.8, 122.4, 117.8, 115.9, 81.3, 62.2; HR-MS (EI) $m / z[M]^{+}$calcd for $\mathrm{C}_{21} \mathrm{H}_{17} \mathrm{NO}_{2}$ 315.1259, found 315.1256 .

( \pm )-cis-3-Phenoxy-4-phenyl-1-(p-tolyl)-2-azetidione

(2b). Colorless crystals, yield $7.9 \mathrm{~g}(60 \%), \mathrm{mp} 175-177^{\circ} \mathrm{C} ; \nu_{\max }(\mathrm{KBr}) /$ $\mathrm{cm}^{-1}$ 3029, 2947, 2856, 1743, 1598, 1494, 1253, 864, 825, 746, $684 ; \delta_{\mathrm{H}}\left(400 \mathrm{MHz}, \mathrm{CDCl}_{3}\right) 7.36(\mathrm{~d}, 2 \mathrm{H}, J 8.4), 7.26(\mathrm{~m}, 4 \mathrm{H}), 7.17(\mathrm{t}$, $2 \mathrm{H}, J$ 8.4), 7.08 (d, 3H, J 7.6), $6.92(\mathrm{t}, 1 \mathrm{H}, J 7.6), 6.79(\mathrm{~d}, 2 \mathrm{H}, J$ 8.4), 5.53 (d, 1H, J 4.8), 5.36 (d, 1H, J 4.8), 2.29 (s, 3H); $\delta_{\mathrm{C}}(100 \mathrm{MHz}$, $\left.\mathrm{CDCl}_{3}\right)$ 163.4, 157.2, 138.7, 137.2, 129.6, 129.4, 129.3, 128.2, 124.7, 122.3, 117.8, 116.0, 81.4, 61.1, 21.4; HR-MS (EI) $m / z[\mathrm{M}]^{+}$ calcd for $\mathrm{C}_{22} \mathrm{H}_{19} \mathrm{NO}_{2} 329.1416$, found 329.1411.

( \pm )-cis-3-Phenoxy-1-( $p$-methoxyphenyl)-4-phenyl-2-azetidinone (2c). Colorless crystals, yield $8.1 \mathrm{~g}$ (60\%), mp $190-191{ }^{\circ} \mathrm{C}$ $\left(186{ }^{\circ} \mathrm{C}\right) ;{ }^{25} \nu_{\max }(\mathrm{KBr}) / \mathrm{cm}^{-1} 3035,2956,2831,1742,1588,1455$, $1441,882,750,696 ; \delta_{\mathrm{H}}\left(600 \mathrm{MHz}, \mathrm{CDCl}_{3}\right) 7.30-7.25(\mathrm{~m}, 7 \mathrm{H}), 7.14$ (m, 2H), 6.90 (t, 1H, J 7.6), 6.80-6.76 (m, 4H), 5.55 (d, 1H, J 4.8), 5.35 (d, $1 \mathrm{H}, J$ 4.8), $3.74(\mathrm{~s}, 3 \mathrm{H}) ; \delta_{\mathrm{C}}\left(125 \mathrm{MHz}, \mathrm{CDCl}_{3}\right)$ 162.2, 157.1, 156.7, 132.8, 130.6, 129.4, 128.9, 128.5, 128.3, 122.3, 119.1, 115.9, 114.6, 81.4, 62.3, 55.6; HR-MS (EI) $m / z[M]^{+}$calcd for $\mathrm{C}_{22} \mathrm{H}_{19} \mathrm{NO}_{3}$ 345.1365 , found 345.1362 .

( \pm )-cis-3-Phenoxy-1-(p-chlorophenyl)-4-phenyl-2-azetidinone (2d). Colorless crystals, yield $3.4 \mathrm{~g}(30 \%), \mathrm{mp} 272-273{ }^{\circ} \mathrm{C}$; $\nu_{\max }(\mathrm{KBr}) / \mathrm{cm}^{-1}$ 3048, 1745, 1598, 1492, 1350, 1251, 866, 837, 746, 684; $\delta_{\mathrm{H}}\left(400 \mathrm{MHz}, \mathrm{DMSO}-\mathrm{d}_{6}\right) 7.39-7.32(\mathrm{~m}, 6 \mathrm{H}), 7.27(\mathrm{~m}$, $3 \mathrm{H}), 7.18(\mathrm{t}, 2 \mathrm{H}, J 7.2), 6.91(\mathrm{t}, 1 \mathrm{H}, J 7.2), 6.80(\mathrm{~d}, 2 \mathrm{H}, J 7.8), 5.78$ (d, $1 \mathrm{H}, J 4.0), 5.70$ (d, $1 \mathrm{H}, J 4.0) ; \delta_{\mathrm{C}}\left(100 \mathrm{MHz}, \mathrm{DMSO}_{6}\right)$ 162.6, 156.2, 135.2, 132.5, 129.1, 128.8, 128.7, 127.9, 127.7, 127.6,
121.5, 118.4, 115.0, 80.6, 60.8; HR-MS (EI) $m / z[\mathrm{M}]^{+}$calcd for $\mathrm{C}_{21} \mathrm{H}_{16}{ }^{35} \mathrm{ClNO}_{2} 349.0870$,found 349.0865.

( \pm )-cis-3-Phenoxy-4-(p-tolyl)-1-phenyl-2-azetidinone $\quad(2 \mathrm{e}){ }^{26}$ Colorless crystals, yield $9.7 \mathrm{~g}(74 \%), \mathrm{mp} 172-173{ }^{\circ} \mathrm{C} ; \nu_{\max }(\mathrm{KBr}) /$ $\mathrm{cm}^{-1}$ 3029, 2947, 2856, 1922, 1746, 1598, 1473, 1388, 746, 684; $\delta_{\mathrm{H}}\left(600 \mathrm{MHz}, \mathrm{CDCl}_{3}\right) 7.36(\mathrm{~d}, 2 \mathrm{H}, J 7.8), 7.26-7.23(\mathrm{~m}, 4 \mathrm{H}), 7.15(\mathrm{t}$, $2 \mathrm{H}, J$ 8.4), 7.06 (d, 3H, J 7.8), 6.90 (t, 1H, J 7.2),6.78 (d, 2H, J 7.8), 5.52 (d, 1H, J 4.8), 5.34 (d, 1H, J 4.8), 2.27 (s, 3H); $\delta_{\mathrm{C}}(125 \mathrm{MHz}$, $\left.\mathrm{CDCl}_{3}\right)$ 163.4, 157.2, 138.7, 137.1, 129.6, 129.4, 129.3, 128.2, 124.7, 122.3, 117.8, 116.0, 81.4, 62.1, 21.4; HR-MS (EI) $m / z[\mathrm{M}]^{+}$ calcd for $\mathrm{C}_{22} \mathrm{H}_{19} \mathrm{NO}_{2} 329.1416$, found 329.1410 .

( \pm )-cis-3-Phenoxy-4-( $p$-methoxyphenyl)-1-phenyl-2-azetidinone (2f). Colorless crystals, yield $8.4 \mathrm{~g}$ (61\%), mp 152-155 ${ }^{\circ} \mathrm{C}$ $\left(150{ }^{\circ} \mathrm{C}\right) ;{ }^{25} \nu_{\max }(\mathrm{KBr}) / \mathrm{cm}^{-1} 3041,2957,2872,1758,1597,1458$, $1384,1362,1251,895,754,690 ; \delta_{\mathrm{H}}\left(400 \mathrm{MHz} \mathrm{CDCl}_{3}\right) 7.36(\mathrm{~d}, 2 \mathrm{H}, J$ 7.6), 7.36-7.25 (m, 4H), $7.17(\mathrm{t}, 2 \mathrm{H}, J 7.6), 7.08(\mathrm{t}, 1 \mathrm{H}, J 7.6), 6.92(\mathrm{t}$, $1 \mathrm{H}, J$ 7.2), 6.80 (m, 4H), 5.53 (d, 1H, J 4.8), 5.35 (d, 1H, J 4.8), 3.75 $(\mathrm{s}, 3 \mathrm{H}) ; \delta_{\mathrm{C}}\left(100 \mathrm{MHz}, \mathrm{CDCl}_{3}\right)$ 163.3, 160.0, 157.2, 137.3, 129.6, 129.4, 129.3, 124.7, 124.5, 122.3, 117.8, 115.9, 114.1, 81.4, 61.9, 55.4; HR-MS (EI) $m / z[\mathrm{M}]^{+}$calcd for $\mathrm{C}_{22} \mathrm{H}_{19} \mathrm{NO}_{3} 345.1365$, found 345.1361 .

( \pm )-cis-3-Phenoxy-4-( $p$-chlorophenyl)-1-phenyl-2-azetidinone (2g). ${ }^{27}$ Colorless crystals, yield $6.5 \mathrm{~g}$ (58\%), mp 193-196 ${ }^{\circ} \mathrm{C}$; $\nu_{\max }(\mathrm{KBr}) / \mathrm{cm}^{-1} 3047,1743,1598,1490,1350,1251,865,837$, 744 and 684; $\delta_{\mathrm{H}}\left(400 \mathrm{MHz}, \mathrm{CDCl}_{3}\right) 7.26-7.11(\mathrm{~m}, 8 \mathrm{H}), 7.08(\mathrm{t}, 2 \mathrm{H}$, $J$ 8.0), $7.01(\mathrm{t}, 1 \mathrm{H}, J$ 7.6), $6.84(\mathrm{t}, 1 \mathrm{H}, J 7.2), 6.68$ (d, 2H, J 8.0), 5.47 (d, $1 \mathrm{H}, J$ 4.8), 5.28 (d, 1H, $J$ 4.8); $\delta_{\mathrm{C}}\left(100 \mathrm{MHz}, \mathrm{CDCl}_{3}\right)$ 163.0, 156.9, 136.9, 134.8, 131.4, 129.65, 129.60, 129.4, 128.9, 125.0, 122.6, 117.7, 115.8, 81.2, 61.5; HR-MS (EI) $m / z[\mathrm{M}]^{+}$calcd for $\mathrm{C}_{21} \mathrm{H}_{16}{ }^{35} \mathrm{ClNO}_{2}$ 349.0870, found 349.0867.

( \pm )-trans-3-Phenoxy-1-( -chlorophenyl)-4-phenyl-2-azetidinone (3d). Colorless crystals, yield $5.1 \mathrm{~g}$ (45\%), mp 149-150 ${ }^{\circ} \mathrm{C}$; $\nu_{\max }(\mathrm{KBr}) / \mathrm{cm}^{-1} 3050,1745,1599,1490,1353,1250,866,838,746$, $685 ; \delta_{\mathrm{H}}\left(600 \mathrm{MHz}, \mathrm{CDCl}_{3}\right) 7.39-7.19(\mathrm{~m}, 11 \mathrm{H}), 7.00(\mathrm{t}, 1 \mathrm{H}, J$ 7.2), $6.87(\mathrm{~d}, 2 \mathrm{H}, J 7.8), 5.11$ (d, $1 \mathrm{H}, J 1.8), 5.00(\mathrm{~d}, 1 \mathrm{H}, J 1.8) ; \delta_{\mathrm{C}}(125 \mathrm{MHz}$, $\mathrm{CDCl}_{3}$ ) 162.7, 157.0, 135.5, 135.2, 129.9, 129.8, 129.6, 129.5, 129.4, 126.5, 122.5, 118.9, 115.5, 87.6, 64.3; HR-MS (EI) $m / z[\mathrm{M}]^{+}$calcd for $\mathrm{C}_{21} \mathrm{H}_{16}{ }^{35} \mathrm{ClNO}_{2}$ 349.0870, found 349.0870.

\subsection{Synthesis of thio- $\beta$-lactams $4 \mathrm{a}-\mathrm{g}$ : general procedure}

A mixture of appropriate $\beta$-lactams $2 \mathbf{a}-\mathbf{g}(4 \mathrm{mmol})$ and Lawesson's reagent $(1.6 \mathrm{~g}, 4 \mathrm{mmol})$ in dry toluene $(15 \mathrm{~mL})$ was refluxed for $3 \mathrm{~h}$. The solvent was then removed in vacuo and the resulting solid was recrystallised from ethanol to give the corresponding thio- $\beta$-lactams $\mathbf{4 a - g}$ in $65-77 \%$ yield.

$( \pm)$-cis-3-Phenoxy-1,4-diphenyazetidin-2-thione (4a). Colorless crystals, $0.88 \mathrm{~g}(67 \%), \mathrm{mp} 170-175^{\circ} \mathrm{C} ; \nu_{\max }(\mathrm{KBr}) / \mathrm{cm}^{-1} 3026$, 3006, 2927, 1589, 1494, 1427, 1228, 1205, 1074, 765, 746, 686; $\delta_{\mathrm{H}}\left(400 \mathrm{MHz}, \mathrm{CDCl}_{3}\right) 7.92(\mathrm{~d}, 2 \mathrm{H}, J 8.4), 7.36-7.13(\mathrm{~m}, 10 \mathrm{H}), 6.93$ (t, 1H, J 6.7), 6.78 (d, 2H, J 8.0), 5.89 (d, 1H, J 4.8), 5.35 (d, 1H, J 4.8); $\delta_{\mathrm{C}}\left(100 \mathrm{MHz}, \mathrm{CDCl}_{3}\right)$ 195.2, 157.2, 138.0, 132.0, 129.4, 129.2, 128.7, 128.3 126.6, 122.5, 118.5, 116.3, 79.5, 70.4; HR-MS (EI) m/ $z[\mathrm{M}]^{+}$calcd for $\mathrm{C}_{21} \mathrm{H}_{17} \mathrm{NOS} 331.1031$, found 331.1028.

( \pm )-cis-3-Phenoxy-1-(p-tolyl)-4-phenylazetidin-2-thione (4b). Colorless crystals, $0.95 \mathrm{~g}(69 \%), 157-159{ }^{\circ} \mathrm{C} ; \nu_{\max }(\mathrm{KBr}) / \mathrm{cm}^{-1}$ 2916, 2831, 2360, 1579, 1404, 1346, 1207, 750, 688; $\delta_{\mathrm{H}}(400 \mathrm{MHz}$, 
$\left.\mathrm{CDCl}_{3}\right) 7.92(\mathrm{~d}, 2 \mathrm{H}, J$ 8.4), $7.32(\mathrm{t}, 2 \mathrm{H} J$ 8.4), 7.26-7.14 (m, 5H), 7.07 (d, 2H J 8.0), 6.92 (t, 1H J 7.6), 6.81 (d, 2H J 8.0), 5.86 (d, 1H, 4.4), $5.32(\mathrm{~d}, 1 \mathrm{H}, J 4.4), 2.28(\mathrm{~s}, 3 \mathrm{H}) ; \delta_{\mathrm{C}}\left(100 \mathrm{MHz}, \mathrm{CDCl}_{3}\right)$ 195.3, 157.3, 139.1, 138.0, 129.45, 129.41, 129.2, 128.9, 128.2, 126.6, 122.4, 118.6, 116.3, 79.7, 70.5, 21.4; HR-MS (EI) $m / z[\mathrm{M}]^{+}$calcd for $\mathrm{C}_{22} \mathrm{H}_{19} \mathrm{NOS} 345.1187$, found 345.1181.

( \pm )-cis-3-Phenoxy-1-( $p$-methoxyphenyl)-4-phenylazetidin-2thione (4c). Colorless crystals, $1.03 \mathrm{~g}(71 \%), \mathrm{mp} 148-150{ }^{\circ} \mathrm{C}$; $\nu_{\max }(\mathrm{KBr}) / \mathrm{cm}^{-1}$ 3031, 2954, 2923, 2835, 1585, 1446, 1438, $1259,1176,1101,850,748,723,684 ; \delta_{\mathrm{H}}\left(400 \mathrm{MHz}, \mathrm{CDCl}_{3}\right) 7.88$ $(\mathrm{d}, 2 \mathrm{H}, J$ 8.0), 7.35-7.26 (m, 5H), $7.14(\mathrm{t}, 2 \mathrm{H}, J 7.2), 6.92(\mathrm{t}, 1 \mathrm{H}, J$ 7.2), 6.84 (d, 2H, J 7.4), 6.79 (d, 2H, J 8.0), 5.84 (d, 1H, J 4.4), $5.35\left(\mathrm{~d}, 1 \mathrm{H}, J\right.$ 4.4), $3.77(\mathrm{~s}, 3 \mathrm{H}) ; \delta_{\mathrm{C}}\left(100 \mathrm{MHz}, \mathrm{CDCl}_{3}\right)$ 193.3, $157.9,157.2$, 132.0, 131.5, 129.3, 129.2, 128.6, 128.3, 122.4, 120.2, 116.2, 114.3, 79.5, 70.6, 55.6; HR-MS (EI) $m / z[\mathrm{M}]^{+}$calcd for $\mathrm{C}_{22} \mathrm{H}_{19} \mathrm{NO}_{2} \mathrm{~S} 361.1136$, found 361.1133.

( \pm )-cis-3-Phenoxy-1-( -chlorophenyl)-4-phenyl-2-azetidin-2thione (4d). Colorless crystals, $1.12 \mathrm{~g}$ (77\%), mp 217-218 ${ }^{\circ} \mathrm{C}$; $\nu_{\max }(\mathrm{KBr}) / \mathrm{cm}^{-1} 3030,2927,1582,1494,1427,1230,1200,1077$, 765, 750, 686; $\delta_{\mathrm{H}}\left(600 \mathrm{MHz}, \mathrm{DMSO}_{\mathrm{d}}\right) 7.93(\mathrm{~d}, 2 \mathrm{H}, J$ 7.8), 7.49 (d, 2H, J 7.8),7.31-7.16 (m, 7H) 6.90 (t, 1H, J 7.8), 6.80 (d, 2H, J 7.8), 6.36 (d, 1H, J 4.2), 5.64 (d, $1 \mathrm{H}, J 4.2) ; \delta_{\mathrm{C}}(125 \mathrm{MHz}$, DMSO$\left.d_{6}\right)$ 195.2, 156.3, 136.0, 131.8130.1, 129.3, 129.2, 128.7, 128.3, 125.3, 121.9, 119.8, 115.2, 78.8, 69.5; HR-MS (EI) $m / z[\mathrm{M}]^{+}$calcd for $\mathrm{C}_{21} \mathrm{H}_{16}{ }^{35} \mathrm{ClNOS} 365.0641$, found 365.0635 .

( \pm )-cis-3-Phenoxy-4-(p-tolyl)-1-phenyl-2-azetidin-2-thione (4e). Colorless crystals, $0.89 \mathrm{~g}$ (65\%), $152-153{ }^{\circ} \mathrm{C} ; \nu_{\max }(\mathrm{KBr}) / \mathrm{cm}^{-1}$ 2916, 2831, 2360, 2343, 1589, 1404, 1346, 1207, 750, 688; $\delta_{\mathrm{H}}(600$ $\left.\mathrm{MHz} \mathrm{CDCl}_{3}\right) 7.90$ (d, 2H, J 7.8), 7.28 (t, 2H, $J$ 7.8), 7.20 (d, 2H, 7.4), $7.15(\mathrm{~m}, 3 \mathrm{H}), 7.03(\mathrm{~d}, 2 \mathrm{H}, J 7.8), 6.88(\mathrm{t}, 1 \mathrm{H}, J 7.8), 6.79(\mathrm{~d}, 2 \mathrm{H}$, $J$ 7.8), 5.83 (d, 1H, J 4.2), 5.28 (d, 1H, J 4.2), 2.25 (s, 3H); $\delta_{\mathrm{C}}(125$ $\mathrm{MHz}, \mathrm{CDCl}_{3}$ ) 195.2, 157.3, 139.0, 138.0, 129.38, 129.36, 129.1, 128.8, 128.2, 126.5, 122.4, 118.5, 116.3, 79.6, 70.4, 21.3; HR-MS (EI) $m / z[\mathrm{M}]^{+}$calcd for $\mathrm{C}_{22} \mathrm{H}_{19} \mathrm{NOS} 345.1187$, found 345.1184.

( \pm )-cis-3-Phenoxy-4-( $\boldsymbol{p}$-methoxyphenyl)-1-phenyl-2-azetidin-2thione (4f). Colorless crystals, $1.05 \mathrm{~g}$ (73\%), mp $148-150{ }^{\circ} \mathrm{C}$; $\nu_{\max }(\mathrm{KBr}) / \mathrm{cm}^{-1} 3030,2953,2923,2835,1586,1446,1438,1259$, $1176,1101,851,750,725,683 ; \delta_{\mathrm{H}}\left(600 \mathrm{MHz} \mathrm{CDCl}_{3}\right) 7.93(\mathrm{~d}, 2 \mathrm{H}, J$ 8.0), 7.34-7.14 (m, 7H), $6.92(\mathrm{t}, 1 \mathrm{H}, J 7.6), 6.81$ (t, 4H, J 7.6), 5.85 $(\mathrm{d}, 1 \mathrm{H}, J 4.4), 5.30(\mathrm{~d}, 1 \mathrm{H}, J 4.4), 3.73(\mathrm{~s}, 3 \mathrm{H}) ; \delta_{\mathrm{C}}\left(125 \mathrm{MHz}, \mathrm{CDCl}_{3}\right)$ $195.1,160.2$, 157.2, 137.9, 129.6, 129.3, 129.1, 126.5, 123.7, 122.3, 118.6, 116.2, 114.0, 79.6, 70.1, 55.3; HR-MS (EI) $m / z[\mathrm{M}]^{+}$ calcd for $\mathrm{C}_{22} \mathrm{H}_{19} \mathrm{NO}_{2} \mathrm{~S} 361.1136$, found 361.1130.

( \pm )-cis-3-Phenoxy-4-(p-chlorophenyl)-1-phenyl-2-azetidin-2thione (4g). Colorless crystals, $1.05 \mathrm{~g}(72 \%), \operatorname{mp~} 166-167{ }^{\circ} \mathrm{C}$; $\nu_{\max }(\mathrm{KBr}) / \mathrm{cm}^{-1} 3033,2927,1587,1494,1425,1230,1212,1077$, 765, 749, 686; $\delta_{\mathrm{H}}\left(400 \mathrm{MHz}, \mathrm{CDCl}_{3}\right) 7.90(\mathrm{~d}, 2 \mathrm{H}, J$ 8.4), 7.35-7.15 (m, 9H), 6.94 (t, 1H, J 7.6), 6.80 (d, 2H, J 8.0), 5.87 (d, 1H, J 4.8), $5.33(\mathrm{~d}, 1 \mathrm{H}, J 4.8) ; \delta_{\mathrm{C}}\left(100 \mathrm{MHz}, \mathrm{CDCl}_{3}\right)$ 195.0, 157.0, 137.7, 135.1, 130.6, 129.6, 129.5, 129.3, 128.9, 126.8, 122.6, 118.4, 116.1, 79.3, 69.6; HR-MS (EI) $m / z[\mathrm{M}]^{+}$calcd for $\mathrm{C}_{21} \mathrm{H}_{16}{ }^{35} \mathrm{CINOS} 365.0641$, found 365.0635 .

\subsection{Synthesis of $\boldsymbol{\beta}$-lactams 8a-e: general procedure}

To a stirred cold $\left(0{ }^{\circ} \mathrm{C}\right)$ solution of appropriate imines 1a-e (10 mmol, 1 equiv.) and triethylamine (25 mmol, 2.5 equiv.) in dry
$\mathrm{CH}_{2} \mathrm{Cl}_{2}(15 \mathrm{~mL})$ under nitrogen atmosphere was added dropwise with a syringe a solution of allyloxyacetyl chloride (25 mmol, 2.5 equiv.) in dry $\mathrm{CH}_{2} \mathrm{Cl}_{2}(5 \mathrm{~mL})$. The mixture was stirred at $0{ }^{\circ} \mathrm{C}$ for $30 \mathrm{~min}$, and then left stirring at room temperature overnight. The reaction mixture was washed with water, $\mathrm{NaHCO}_{3}$ solution and brine. The organic layer was then dried over anhydrous $\mathrm{Na}_{2} \mathrm{SO}_{4}$ and the solvent was removed in vacuo. The remaining solid was recrystallized from ethanol to give corresponding $\beta$-lactams $\mathbf{8 a}-\mathbf{e}$ as colourless solid in $69-84 \%$ yield.

( \pm )-cis-3-Allyloxy-1,4-diphenyl-2-azetidinone (8a). Colorless crystals, yield $2.10 \mathrm{~g}$ (75\%), mp $131-133{ }^{\circ} \mathrm{C} ; \nu_{\max }(\mathrm{KBr}) / \mathrm{cm}^{-1}$ 3036, 2910, 2857, 1741, 1595, 1497, 1389, 1184, 1132, 926, 752; $\delta_{\mathrm{H}}\left(400 \mathrm{MHz}, \mathrm{CDCl}_{3}\right) 7.42-7.23(\mathrm{~m}, 7 \mathrm{H}), 7.26(\mathrm{t}, 2 \mathrm{H}, J 7.6), 7.05(\mathrm{t}$, $1 \mathrm{H}, J$ 7.6), 5.57 (m, 1H), 5.19 (d, 1H, J 4.8, H-4), 5.08 (dd, 1H, $J$ 8.8, 1.2), 5.00 (dd, 1H, J 18.2, 1.2), 4.96 (d, 1H, J 4.8, H-3), 3.89 (dd, $1 \mathrm{H}, J 12.4,5.6), 3.74$ (dd, $1 \mathrm{H}, J 12.4,5.6) ; \delta_{\mathrm{C}}(100 \mathrm{MHz}$, $\mathrm{CDCl}_{3}$ ) 164.6, 137.2, 133.6, 133.3, 129.2, 128.7, 128.6, 128.2, 124.5, 118.2, 117.6, 82.7, 71.6, 62.1; HR-MS (EI) $\mathrm{m} / \mathrm{z}[\mathrm{M}]^{+}$calcd for $\mathrm{C}_{18} \mathrm{H}_{17} \mathrm{NO}_{2} 279.1259$, found 279.1253; anal. calcd: $\mathrm{C} 77.40, \mathrm{H}$ 6.13, N 5.01. Found C 77.20, H 6.08, N 4.90.

( \pm )-trans-3-Allyloxy-1,4-diphenyl-2-azetidinone (9a). Colorless crystals, yield $0.2 \mathrm{~g}$ (4\%), mp 94-96 ${ }^{\circ} \mathrm{C} ; \nu_{\max }(\mathrm{KBr}) / \mathrm{cm}^{-1}$ 3035, 2910, 2857, 1740, 1595, 1497, 1390, 1184, 1133, 926, 752; $\delta_{\mathrm{H}}\left(400 \mathrm{MHz}, \mathrm{CDCl}_{3}\right) 7.41-7.24(\mathrm{~m}, 9 \mathrm{H}), 7.07(\mathrm{t}, 1 \mathrm{H}, J$ 7.8), 5.95 (m, 1H), 5.32 (dd, 1H, J 15.6, 1.6), 5.23 (dd, 1H, J 10.4, 1.2), 4.95 (d, 1H, J 2.4, H-4), 4.45 (d, 1H, J 2.4, H-3), 4.30 (dd, 1H, J 12.4, 5.6), 4.24 (dd, $1 \mathrm{H}, J$ 12.4, 5.6); HR-MS (EI) $m / z[\mathrm{M}]^{+}$calcd for $\mathrm{C}_{18} \mathrm{H}_{17} \mathrm{NO}_{2} 279.1259$, found 279.1254; anal. calcd: $\mathrm{C} 77.40, \mathrm{H}$ 6.13, N 5.01. Found C 77.18, H 6.09, N 4.93.

$( \pm)$-cis-3-Allyloxy-1-( $\boldsymbol{p}$-methoxyphenyl)-4-phenyl-2-azetidinone (8b). Colorless crystals, yield $2.60 \mathrm{~g}$ (84\%), mp 120-121 ${ }^{\circ} \mathrm{C}$; $\nu_{\max }(\mathrm{KBr}) / \mathrm{cm}^{-1} 3036,2977,2837,1741,1595,1690,1514,1243$, $1185,1115,993,829,694 ; \delta_{\mathrm{H}}\left(400 \mathrm{MHz}, \mathrm{CDCl}_{3}\right) 7.39-7.28(\mathrm{~m}, 7 \mathrm{H})$, 6.80-6.78 (m, 2H) 5.59 (m, 1H), 5.17 (d, 1H, J 4.8, H-4), 5.08 (dd, $1 \mathrm{H}, J$ 8.8, 1.2), 5.04 (dd, 1H, J 18.2, 1.2), 4.97 (d, 1H, J 4.8, H-3), 3.89 (dd, $1 \mathrm{H}, J 12.2$, 5.6), 3.76 (dd, $1 \mathrm{H}, J 12.2,5.6), 3.73$ (s, $3 \mathrm{H}$, $\left.\mathrm{OCH}_{3}\right) ; \delta_{\mathrm{C}}\left(100 \mathrm{MHz}, \mathrm{CDCl}_{3}\right) 164.0,156.5,133.7,133.4,130.8$, 128.7, 128.6, 128.3, 118.9, 118.1, 114.5, 82.9, 71.6, 62.2 , 55.6; HRMS (EI) $m / z[\mathrm{M}]^{+}$calcd for $\mathrm{C}_{19} \mathrm{H}_{19} \mathrm{NO}_{3} 309.1365$, found 309.1359; anal. calcd: C 73.77, H 6.19, N 4.53. Found C 73.49, H 6.00, N 4.34.

( \pm )-cis-3-Allyloxy-1-(p-chlorophenyl)-4-phenyl-2-azetidinone (8c). Colorless crystals, yield $2.15 \mathrm{~g}$ (69\%), mp 98-100 ${ }^{\circ} \mathrm{C}$; $\nu_{\max }(\mathrm{KBr}) / \mathrm{cm}^{-1} 3065,3037,2961,2903,2857,1741,1595,1496$, $1385,1176,1131,839,814 ; \delta_{\mathrm{H}}\left(400 \mathrm{MHz}, \mathrm{CDCl}_{3}\right) 7.37-7.18(\mathrm{~m}$, 9H), 5.55 (m, 1H), 5.17 (d, 1H, J 4.8, H-4), 5.07 (dd, 1H, J 9.6, 1.2), 5.03 (dd, 1H, J 18.4, 1.2), 4.97 (d, 1H, J 4.8, H-3), 3.88 (dd, $1 \mathrm{H}, J 12.4,5.6), 3.74(\mathrm{dd}, 1 \mathrm{H}, J 12.4,5.6) ; \delta_{\mathrm{C}}\left(100 \mathrm{MHz}, \mathrm{CDCl}_{3}\right)$ $164.5,135.8,133.3,133.1,129.6$, 129.3, 129.0, 128.8, 128.2, 118.9, 118.4, 83.0, 71.8, 62.3; HR-MS (EI) $\mathrm{m} / z[\mathrm{M}]^{+}$calcd for $\mathrm{C}_{18} \mathrm{H}_{16} \mathrm{ClNO}_{2} 313.0870$, found 313.0864; anal. calcd: C 68.90, H 5.14, N 4.46. Found C 68.71, H 5.08, N 4.32.

$( \pm)$-cis-3-Allyloxy-4-( $p$-methoxyphenyl)-1-phenyl-2-azetidinone (8d). Colorless crystals, yield $2.55 \mathrm{~g}$ (82\%), mp 90-91 ${ }^{\circ} \mathrm{C}$; $\nu_{\max }(\mathrm{KBr}) / \mathrm{cm}^{-1} 3037,2960,2898,1749,1615,1510,1393,1247$, $1176,1131,833,752 ; \delta_{\mathrm{H}}\left(400 \mathrm{MHz}, \mathrm{CDCl}_{3}\right) 7.33$ (d, 4H, J 6.8), 7.24 
(t, 2H, J 7.2), $7.05(\mathrm{t}, 1 \mathrm{H}, J$ 7.2), $6.89(\mathrm{~d}, 2 \mathrm{H}, J 6.8), 5.59(\mathrm{~m}, 1 \mathrm{H})$, 5.14 (d, 1H, J 4.8, H-4), 5.11 (dd, $1 \mathrm{H}, J$ 8.8, 1.2), 5.07 (dd, $1 \mathrm{H}, J$ 18.2 1.2), 4.92 (d, 1H, J 4.8, H-3), 3.89 (dd, 1H, J 12.4, 5.6), 3.80 (s, $\left.3 \mathrm{H}, \mathrm{OCH}_{3}\right), 3.76(\mathrm{dd}, 1 \mathrm{H}, J 12.4,5.6) ; \delta_{\mathrm{C}}\left(100 \mathrm{MHz}, \mathrm{CDCl}_{3}\right)$ 164.7, 160.0, 137.3, 133.5, 129.5, 129.2, 125.4, 124.4, 118.2, 117.7, 114.1, 82.7, 71.7, 61.7, 55.4; HR-MS (EI) $m / z[M]^{+}$calcd for $\mathrm{C}_{19} \mathrm{H}_{19} \mathrm{NO}_{3}$ 309.1365, found 309.1359; anal. calcd: C 73.77, H 6.19, N 4.53. Found C 73.40, H 6.05, N 4.54.

( \pm )-cis-3-Allyloxy-4-(p-chlorophenyl)-1-phenyl-2-azetidinone (8e). Colorless crystals, yield $2.19 \mathrm{~g}$ (70\%), mp 109-110 ${ }^{\circ} \mathrm{C}$; $\nu_{\max }(\mathrm{KBr}) / \mathrm{cm}^{-1}$ 3062, 3030, 2983, 2905, 1756, 1597, 1497, $1387,1125,1087,929,750,685 ; \delta_{\mathrm{H}}\left(400 \mathrm{MHz}, \mathrm{CDCl}_{3}\right) 7.37-7.23$ $(\mathrm{m}, 8 \mathrm{H}), 7.07(\mathrm{t}, 1 \mathrm{H}, J 7.8), 5.60(\mathrm{~m}, 1 \mathrm{H}), 5.17(\mathrm{~d}, 1 \mathrm{H}, J 4.8, \mathrm{H}-4)$, 5.08 (dd, 1H, J 9.6, 1.2), 5.06 (dd, 1H, J 18.4, 1.2), 4.95 (d, 1H, $J$ 4.8, H-3), 3.93 (dd, 1H, J 12.4, 5.6), 3.78 (dd, $1 \mathrm{H}, J 12.4,5.6$ ); $\delta_{\mathrm{C}}\left(100 \mathrm{MHz}, \mathrm{CDCl}_{3}\right) 164.5,137.0,134.7,133.2,132.3,129.6$, 129.3, 129.0, 124.7, 118.4, 117.5, 82.7, 71.9, 61.4; HR-MS (EI) $m / z[\mathrm{M}]^{+}$calcd for $\mathrm{C}_{18} \mathrm{H}_{16}{ }^{35} \mathrm{ClNO}_{2}$ 313.0870, found 313.0863; anal. calcd: C 68.90, H 5.14, N 4.46. Found C 68.69, H 5.04, N 4.38.

\subsection{Synthesis of thio- $\beta$-lactams 10a-e: general procedure}

A mixture of appropriate $\beta$-lactams 1a-e $(2 \mathrm{mmol})$ and Lawesson's reagent ( $0.8 \mathrm{~g}, 2 \mathrm{mmol}, 2$ equiv.) in dry toluene $(15 \mathrm{~mL})$ was refluxed for $3 \mathrm{~h}$. Then the solvent evaporated at reduced pressure. Finally, the resulting solid was recrystallised in ethanol to give thio- $\beta$-lactams 10a-e in $65-76 \%$ yield.

( \pm )-cis-3-Allyloxy-1,4-diphenylazetidin-2-thione (10a). Colorless crystals, yield $0.40 \mathrm{~g}(70 \%), \mathrm{mp} 70-72{ }^{\circ} \mathrm{C} ; \nu_{\max }(\mathrm{KBr}) / \mathrm{cm}^{-1}$ 3067, 3036, 2981, 2920, 1586, 1495, 1497, 1389, 1184, 1132, 926, $752 ; \delta_{\mathrm{H}}\left(400 \mathrm{MHz}, \mathrm{CDCl}_{3}\right) 7.88(\mathrm{~d}, 2 \mathrm{H}, J 8.0), 7.40-7.26(\mathrm{~m}, 7 \mathrm{H})$, 7.17 (t, 1H, J 7.8), 5.71 (d, 1H, J 4.4, H-4),5.58 (m, 1H), 5.10-5.05 (m, 2H), 4.68 (d, 1H, J 4.4, H-3), 4.05 (dd, 1H, J 12.8, 5.6), 3.85 (dd, $1 \mathrm{H}, J$ 12.8, 5.6); $\delta_{\mathrm{C}}\left(100 \mathrm{MHz}, \mathrm{CDCl}_{3}\right)$ 197.5, 138.1, 133.5, 132.8, 129.1, 128.7, 128.3, 126.4, 118.5, 118.1, 80.6, 71.3, 70.4; HR-MS (EI) $m / z[\mathrm{M}]^{+}$calcd for $\mathrm{C}_{18} \mathrm{H}_{17} \mathrm{NOS} 295.1031$, found 295.1024; anal. calcd: C 73.19, H 5.80, N 4.74, S 10.85. Found C 72.89, H 5.57, N 4.61, S 10.64.

( \pm )-cis-3-Allyloxy-1-( -methoxyphenyl)-4-phenylazetidin-2thione (10b). Colorless crystals, yield $0.49 \mathrm{~g}(76 \%), \mathrm{mp} 93-94{ }^{\circ} \mathrm{C}$; $\nu_{\max }(\mathrm{KBr}) / \mathrm{cm}^{-1}$ 3066, 3031, 2955, 2836, 1597, 1510, 1449, 1267, 1175, 1062, 830, 699; $\delta_{\mathrm{H}}\left(400 \mathrm{MHz}, \mathrm{CDCl}_{3}\right) 7.83(\mathrm{~d}, 2 \mathrm{H}, J$ 7.6), 7.37-7.33 (m, 5H), 6.78 (d, 2H, J 7.2), 5.67 (d, 1H, J 4.4, H-4), 5.56 $(\mathrm{m}, 1 \mathrm{H}), 5.07-5.03(\mathrm{~m}, 2 \mathrm{H}), 4.68(\mathrm{~d}, 1 \mathrm{H}, J 4.4, \mathrm{H}-3), 4.00(\mathrm{dd}, 1 \mathrm{H}, J$ 12.8, 5.6), 3.83 (dd, $1 \mathrm{H}, J 12.8,5.6), 3.73\left(\mathrm{~s}, 3 \mathrm{H}, \mathrm{OCH}_{3}\right) ; \delta_{\mathrm{C}}(100$ $\left.\mathrm{MHz}, \mathrm{CDCl}_{3}\right)$ 195.5, 157.6, 133.4, 132.7, 131.6, 129.0, 128.7, 128.3, 120.1, 118.0, 114.1, 80.6, 71.2, 70.5, 55.5; HR-MS (EI) $m / z$ $[\mathrm{M}]^{+}$calcd for $\mathrm{C}_{19} \mathrm{H}_{19} \mathrm{NO}_{2} \mathrm{~S} 325.1136$, found 325.1132; anal. calcd: C 70.12, H 5.88, N 4.30, S 9.85. Found C 69.97, H 5.52, N 4.21, S 9.70 .

( \pm )-cis-3-Allyloxy-1-(p-chlorophenyl)-4-phenylazetidin-2-thione (10c). Colorless crystals, yield $0.42 \mathrm{~g}$ (65\%), mp $34-35{ }^{\circ} \mathrm{C}$; $\nu_{\max }(\mathrm{KBr}) / \mathrm{cm}^{-1}$ 3067, 3031, 2981, 2903, 2864, 1576, 1493, 1399, 1266, 1166, 989, 827; $\delta_{\mathrm{H}}\left(400 \mathrm{MHz}, \mathrm{CDCl}_{3}\right) 7.83$ (d, 2H, J 7.6), 7.36 (m, 5H), 7.22 (d, 2H, J 8.8), 5.69 (d, 1H, J 4.4, H-4), 5.57 (m, 1H), 5.09-5.05 (m, 2H), 4.69 (d, 1H,J 4.4, H-3), 4.03 (dd, 1H,J12.8, 5.6),
3.84 (dd, $1 \mathrm{H}, J 12.8,5.6) ; \delta_{\mathrm{C}}\left(100 \mathrm{MHz}, \mathrm{CDCl}_{3}\right)$ 197.6, 136.5, 133.3, 132.3, 131.2, 129.2, 129.1, 128.8, 128.2, 119.7, 118.2, 80.7, 71.3, 70.5; HR-MS (EI) $\mathrm{m} / z$ [M] $]^{+}$calcd for $\mathrm{C}_{18} \mathrm{H}_{16}{ }^{35} \mathrm{ClNOS} 329.0641$, found 329.0633; anal. calcd: $\mathrm{C} 65.45, \mathrm{H} 4.89, \mathrm{~N} 4.25, \mathrm{~S} 9.72$. Found C 65.19, H 4.69, N 4.11, S 9.62.

$( \pm)$-cis-3-Allyloxy-4-( -methoxyphenyl)-1-phenylazetidin-2thione (10d). Colorless crystals, yield $0.47 \mathrm{~g}$ (72\%), mp 90$91{ }^{\circ} \mathrm{C} ; \nu_{\max }(\mathrm{KBr}) / \mathrm{cm}^{-1}$ 3066, 3032, 2955, 2836, 1594, 1517, $1449,1267,1175,1062,979,699 ; \delta_{\mathrm{H}}\left(400 \mathrm{MHz} \mathrm{CDCl}_{3}\right) 7.88(\mathrm{~d}$, $2 \mathrm{H}, J$ 8.8), $7.32-7.26(\mathrm{~m}, 4 \mathrm{H}), 7.15(\mathrm{t}, 1 \mathrm{H}, J 7.2), 6.88(\mathrm{~d}, 2 \mathrm{H}, J$ 8.8), 5.67 (d, $1 \mathrm{H}, J$ 4.4, H-4),5.63 (m, 1H), 5.14-5.07 (m, $2 \mathrm{H})$, 4.65 (d, 1H, J 4.4, H-3), 4.04 (dd, 1H, J 12.8, 5.6), 3.88 (dd, 1H, J 12.8, 5.6), $3.79\left(\mathrm{~s}, 3 \mathrm{H}, \mathrm{OCH}_{3}\right) ; \delta_{\mathrm{C}}\left(100 \mathrm{MHz}, \mathrm{CDCl}_{3}\right)$ 197.5, 160.2, 138.1, 133.5, 129.7, 129.0, 126.3, 124.5, 118.6, 118.1, 114.2, 80.6, 71.3, 70.1, 55.4; HR-MS (EI) $m / z[\mathrm{M}]^{+}$calcd for $\mathrm{C}_{19} \mathrm{H}_{19} \mathrm{NO}_{2} \mathrm{~S}$ 325.1136, found 325.1132; anal. calcd: C 70.12, H 5.88, N 4.30, S 9.85. Found C 69.89, H 5.58, N 4.23, S 9.69.

( \pm )-cis-3-Allyloxy-4-( -chlorophenyl)-1-phenylazetidin-2-thione (10e). Colorless crystals, yield $0.45 \mathrm{~g}$ (68\%), mp 58-59 ${ }^{\circ} \mathrm{C}$; $\nu_{\max }(\mathrm{KBr}) / \mathrm{cm}^{-1}$ 3069, 3047, 2923, 2857, 1595, 1494, 1426, 1266, 1164, 934, 834; $\delta_{\mathrm{H}}\left(400 \mathrm{MHz}, \mathrm{CDCl}_{3}\right) 7.83$ (d, 2H, J 8.8), 7.36-7.26 (m, 6H), 7.18 (t, 1H, J 7.2), 5.69 (d, 1H, J 4.4,H-4), $5.60(\mathrm{~m}, 1 \mathrm{H})$, 5.14-5.09 (m, 2H), 4.67 (d, 1H, J 4.4, H-3), 4.09 (dd, 1H,J 12.8, 5.6), 3.89 (dd, $1 \mathrm{H}, J 12.8,5.6) ; \delta_{\mathrm{C}}\left(100 \mathrm{MHz}, \mathrm{CDCl}_{3}\right)$ 197.5, 137.8, 135.0, 133.3, 131.5, 129.7, 129.1, 129.0, 126.5, 118.4, 118.3, 80.4, 71.5, 69.6; HR-MS (EI) $\mathrm{m} / z[\mathrm{M}]^{+}$calcd for $\mathrm{C}_{18} \mathrm{H}_{16}{ }^{35} \mathrm{CINOS} 329.0641$, found 329.0636; anal. calcd: C 65.45, H 4.89, N 4.25, S 9.72. Found C 65.21, H 4.76, N 4.14, S 9.53.

\subsection{Pyrolysis product}

(A) Flash vacuum pyrolysis. The apparatus used was similar to the one which has been described in our recent publications. ${ }^{28-30}$ The sample of the substrate was volatilized from a tube in a Büchi Kugelrohr oven through a $30 \times 2.5 \mathrm{~cm}$ horizontal fused quartz tube. This was heated externally by a Carbolite Eurotherm tube furnace MTF-12/38A to a temperature of 550 or $600{ }^{\circ} \mathrm{C}$, the temperature being monitored by $\mathrm{Pt} / \mathrm{Pt}-13 \%$ $\mathrm{Rh}$ thermocouple situated at the center of the furnace. The products were collected in a U-shaped trap cooled in liquid nitrogen. The whole system was maintained at a pressure of $10^{-2}$ Torr by an Edwards Model E2M5 high capacity rotary oil pump, the pressure being measured by a Pirani gauge situated between the cold trap and pump. Under these conditions the contact time in the hot zone was estimated to be $c a .10 \mathrm{~ms}$. The different fractions of the product collected in the U-shaped trap were analyzed by ${ }^{1} \mathrm{H},{ }^{13} \mathrm{C}$ NMR, IR and LC-MS. Relative and percent yields were determined from NMR.

(B) Static pyrolysis. A sample of the substrate ( $1 \mathrm{mmol})$, was introduced in the reaction tube $(1.5 \times 12 \mathrm{~cm}$ Pyrex $)$, cooled in liquid nitrogen, sealed under vacuum ( 0.01 Torr) and placed in the pyrolyzer for 15 minutes at 320 and $270{ }^{\circ} \mathrm{C}$, a temperature that is required for complete pyrolysis of the substrate. The static sealed-tube (STP) pyrolysis was conducted in a custommade Chemical Data System (CDS) pyrolyser consisting of an aluminum block with a groove to accommodate the Pyrex sealed-tube reactor, and fitted with a platinum-resistance 
thermometer and thermocouple connected to a Comark microprocessor thermometer. The block temperature was controlled by a Eurothem 093 precision temperature regulator. Aluminum was chosen for its low temperature gradient and resistance to elevated temperatures.

All the identified products in the STP and FVP gave satisfactory NMR $\left({ }^{1} \mathrm{H},{ }^{13} \mathrm{C}\right)$ and MS., compounds $(E / Z) \mathbf{5 a - d},{ }^{31-35}$ yield $3-25 \%$; compounds $7, \mathbf{1 3 a}-\mathbf{d},{ }^{19,36,37}$ yield $0-24 \%$; compounds 12a-c, ${ }^{17,18}$ yield $12-31 \%$.

(E)-2-Phenyl-1-phenoxyethene $(\mathbf{5 a}) .^{31}$ Colorless oil, $\delta_{\mathrm{H}}(400$ $\mathrm{MHz}, \mathrm{CDCl}_{3}$ ) 7.38-7.21 (m, 9H), 7.09 (d, 2H, $J$ 8.0), 6.35 (d, 1H, 12.4); $\delta_{\mathrm{C}}\left(100 \mathrm{MHz}, \mathrm{CDCl}_{3}\right)$ 157.1, 143.4, 135.1, 129.7, 128.7, 126.7, 125.6, 123.2, 116.9, 113.6; HR-MS (EI) $m / z[\mathrm{M}]^{+}$calcd for $\mathrm{C}_{14} \mathrm{H}_{12} \mathrm{O}$ 196.0888, found 196.0882.

(Z)-2-Phenyl-1-phenoxyethene $(\mathbf{5 a}){ }^{34}$ Colorless oil, $\delta_{\mathrm{H}}(400$ $\mathrm{MHz}, \mathrm{CDCl}_{3}$ ) 7.40-7.10 (m, 10H), 6.63 (d, 1H, J 6.6), 5.64 (d, 1H, 6.6); $\delta_{\mathrm{C}}\left(100 \mathrm{MHz}, \mathrm{CDCl}_{3}\right)$ 157.7, 142.1, 135.4, 130.2, 129.1, 128.7, 127.1, 123.8, 117.4, 110.9; HR-MS (EI) $\mathrm{m} / \mathrm{z}[\mathrm{M}]^{+}$calcd for $\mathrm{C}_{14} \mathrm{H}_{12} \mathrm{O}$ 196.0888, found 196.0885.

(E)-2-p-Tolyl-1-phenoxyethene $(\mathbf{5 b}) .{ }^{31}$ Colorless oil, $\delta_{\mathrm{H}}(400$ $\mathrm{MHz}, \mathrm{CDCl}_{3}$ ) 7.41-7.06 (m, 10H), 6.40 (d, 1H, J 12.4), 2.34 (s, $3 \mathrm{H}) ; \delta_{\mathrm{C}}\left(100 \mathrm{MHz}, \mathrm{CDCl}_{3}\right)$ 157.0, 142.3, 135.8, 132.0, 128.7, 128.6, 125.7, 123.3, 116.8, 113.6, 21.1; HR-MS (EI) $\mathrm{m} / z[\mathrm{M}]^{+}$calcd for $\mathrm{C}_{15} \mathrm{H}_{14} \mathrm{O} 210.1045$, found. 210.1040.

(E)-2-(p-Methoxyphenyl)-1-phenoxyethene (5c). ${ }^{35}$ Pale yellow oil, $\delta_{\mathrm{H}}\left(400 \mathrm{MHz}, \mathrm{CDCl}_{3}\right) 7.61-7.34(\mathrm{~m}, 6 \mathrm{H}), 7.13(\mathrm{~m}, 2 \mathrm{H}), 6.89(\mathrm{~d}$, $2 \mathrm{H}, J$ 8.4), 6.45 (d, $1 \mathrm{H}, J 12.4), 3.93(\mathrm{~s}, 3 \mathrm{H}) ; \delta_{\mathrm{C}}\left(100 \mathrm{MHz}, \mathrm{CDCl}_{3}\right)$ 156.0, 156.4, 133.0, 132.7, 129.3, 128.7, 123.6, 118.4, 114.8, 113.9, 55.4; HR-MS (EI) $m / z[\mathrm{M}]^{+}$calcd for $\mathrm{C}_{15} \mathrm{H}_{14} \mathrm{O}_{2} 226.0994$, found 226.0990 .

(Z)-2-(p-Methoxyphenyl)-1-phenoxyethene $(\mathbf{5 c}) .{ }^{33}$ Pale yellow oil: $\delta_{\mathrm{H}}\left(400 \mathrm{MHz}, \mathrm{CDCl}_{3}\right)$ 7.61-7.34 (m, 5H), $7.12(\mathrm{~m}, 2 \mathrm{H}), 6.87(\mathrm{~d}$, $2 \mathrm{H}, J$ 8.4), 6.48 (d, $1 \mathrm{H}, J$ 6.8), 5.50 (d, 1H, $J$ 6.8), 3.91 (s, 3H); $\delta_{\mathrm{C}}\left(100 \mathrm{MHz}, \mathrm{CDCl}_{3}\right) 156.9,156.2,133.1,132.7,129.2,128.7$, $123.7,118.3,114.8,113.6,55.4$; HR-MS (EI) $\mathrm{m} / z[\mathrm{M}]^{+}$calcd for $\mathrm{C}_{15} \mathrm{H}_{14} \mathrm{O}_{2}$ 226.0994, found 226.0991.

(E)-2-(p-Chlorophenyl)-1-phenoxyethene (5d). ${ }^{32}$ Colorless oil, $\delta_{\mathrm{H}}\left(400 \mathrm{MHz}, \mathrm{CDCl}_{3}\right): 7.36(\mathrm{t}, 2 \mathrm{H}, J$ 8.0), 7.26-7.10 (m, 6H), 7.03 (d, $2 \mathrm{H}, J$ 8.0), 6.32 (d, $1 \mathrm{H}, J$ 12.4); $\delta_{\mathrm{C}}\left(100 \mathrm{MHz}, \mathrm{CDCl}_{3}\right): 157.1$, 144.0, 133.5, 132.0, 129.9, 128.8, 126.6, 123.1, 117.1, 112.0; HRMS (EI) $m / z$ [M] $]^{+}$calcd for $\mathrm{C}_{14} \mathrm{H}_{11}{ }^{35} \mathrm{ClO} 230.0498$, found 230.0498 .

1,3-Diphenylurea (7a). ${ }^{19}$ Colorless solid, mp $241-242{ }^{\circ} \mathrm{C}(240$ $\left.{ }^{\circ} \mathrm{C}\right):{ }^{19} \delta_{\mathrm{H}}(400 \mathrm{MHz}$, DMSO-d 6 ): $8.70(\mathrm{~s}, 2 \mathrm{H}), 7.51-7.22(\mathrm{~m}, 8 \mathrm{H})$, 7.00-6.89 (m, 2H); $\delta_{\mathrm{C}}\left(100 \mathrm{MHz}, \mathrm{DMSO}_{\mathrm{d}}\right)$ : 152.3, 139.6, 128.3, 121.9, 118.0; HR-MS (EI) $m / z[\mathrm{M}]^{+}$calcd for $\mathrm{C}_{13} \mathrm{H}_{12} \mathrm{~N}_{2} \mathrm{O}$ 212.0950, found 212.0950 .

1,3-Bis-(p-tolyl)urea (7b). ${ }^{19}$ Colorless solid, mp 262-264 ${ }^{\circ} \mathrm{C}$ $\left(268{ }^{\circ} \mathrm{C}\right):{ }^{19} \delta_{\mathrm{H}}\left(400 \mathrm{MHz}, \mathrm{DMSO}-\mathrm{d}_{6}\right): 8.56(\mathrm{~s}, 2 \mathrm{H}), 7.33(\mathrm{~d}, 4 \mathrm{H}, J$ 8.0), 7.05 (d, 4H, $J$ 8.0), $2.24(\mathrm{~s}, 6 \mathrm{H}) ; \delta_{\mathrm{C}}\left(100 \mathrm{MHz}, \mathrm{DMSO}_{6} \mathrm{~d}_{6}\right)$ : 157.3, 142.6, 135.3, 134.9, 123.0, 24.9; HR-MS (EI) $m / z[\mathrm{M}]^{+}$calcd for $\mathrm{C}_{15} \mathrm{H}_{16} \mathrm{~N}_{2} \mathrm{O} 240.1263$, found 240.1260 .

1,3-Bis-(p-methoxyphenyl)urea (7c). ${ }^{19}$ Colorless solid, mp 241$242{ }^{\circ} \mathrm{C}\left(242{ }^{\circ} \mathrm{C}\right):{ }^{19} \delta_{\mathrm{H}}\left(400 \mathrm{MHz}, \mathrm{DMSO}-\mathrm{d}_{6}\right): 8.65(\mathrm{~s}, 2 \mathrm{H}), 7.43(\mathrm{~d}$, $4 \mathrm{H}, J$ 8.6), 6.85 (d, $4 \mathrm{H}, J$ 8.6), 3.73 (s, 6H); $\delta_{\mathrm{C}}(100 \mathrm{MHz}$, DMSO$\mathrm{d}_{6}$ ): 159.3, 158.6, 138.3, 125.9, 119.0, 59.9; HR-MS (EI) $m / z[\mathrm{M}]^{+}$ calcd for $\mathrm{C}_{15} \mathrm{H}_{16} \mathrm{~N}_{2} \mathrm{O}_{3} 272.1161$, found 272.1169.
1,3-Bis-(p-chlorophenyl)urea (7d). ${ }^{19}$ Colorless solid, mp 297$299{ }^{\circ} \mathrm{C}\left(301{ }^{\circ} \mathrm{C}\right)::^{19} \delta_{\mathrm{H}}\left(400 \mathrm{MHz}, \mathrm{DMSO}-\mathrm{d}_{6}\right): 8.85(\mathrm{~s}, 2 \mathrm{H}), 7.41(\mathrm{~d}$, $4 \mathrm{H}, J$ 8.6), 7.34 (d, $4 \mathrm{H}, J$ 8.6); $\delta_{\mathrm{C}}\left(100 \mathrm{MHz}, \mathrm{DMSO}_{6}\right):$ 157.3, 143.6, 133.3, 130.5, 125.3; HR-MS (EI) $\mathrm{m} / z[\mathrm{M}]^{+}$calcd for $\mathrm{C}_{13} \mathrm{H}_{10}{ }^{35} \mathrm{Cl}_{2} \mathrm{~N}_{2} \mathrm{O} 280.0170$, found 280.0167.

1,3-Diphenylthiourea (13a). ${ }^{36}$ Colorless solid, mp $150-151{ }^{\circ} \mathrm{C}$ $\left(154{ }^{\circ} \mathrm{C}\right): \delta_{\mathrm{H}}\left(400 \mathrm{MHz}, \mathrm{CDCl}_{3}\right): 8.30(\mathrm{~s}, 2 \mathrm{H}), 7.41-7.27(\mathrm{~m}, 10 \mathrm{H})$; $\delta_{\mathrm{C}}\left(100 \mathrm{MHz}, \mathrm{CDCl}_{3}\right): 180.1,137.6,129.5,127.1$, 125.5; HR-MS (EI) $m / z[\mathrm{M}]^{+}$calcd for $\mathrm{C}_{13} \mathrm{H}_{12} \mathrm{~N}_{2} \mathrm{~S} 228.0721$, found 228.0718 .

1,3-Bis-(p-tolyl)thiourea (13b). ${ }^{36}$ Colorless solid, mp 178-180 ${ }^{\circ} \mathrm{C}\left(179{ }^{\circ} \mathrm{C}\right): \delta_{\mathrm{H}}\left(400 \mathrm{MHz}, \mathrm{DMSO}-\mathrm{d}_{6}\right): 9.56(\mathrm{~s}, 2 \mathrm{H}), 7.34-7.14(\mathrm{~m}$, $8 \mathrm{H}), 2.31(\mathrm{~s}, 6 \mathrm{H}) ; \delta_{\mathrm{C}}\left(100 \mathrm{MHz}, \mathrm{DMSO}-\mathrm{d}_{6}\right): 181.2,137.6,134.3$, 130.7, 125.8, 21.9; HR-MS (EI) $m / z[\mathrm{M}]^{+}$calcd for $\mathrm{C}_{15} \mathrm{H}_{16} \mathrm{~N}_{2} \mathrm{~S}$ 256.1034, found 256.1031 .

1,3-Bis-(p-methoxyphenyl)thiourea (13c) $\cdot{ }^{37}$ Colorless solid, mp $184-185{ }^{\circ} \mathrm{C}\left(200{ }^{\circ} \mathrm{C}\right): \delta_{\mathrm{H}}\left(400 \mathrm{MHz}, \mathrm{DMSO}_{6}\right): 9.54(\mathrm{~s}, 2 \mathrm{H}), 7.31$ (d, $4 \mathrm{H}, J$ 8.8), 6.85 (d, 4H, J 8.8), $3.72(\mathrm{~s}, 6 \mathrm{H}) ; \delta_{\mathrm{C}}(100 \mathrm{MHz}$, DMSO$\mathrm{d}_{6}$ ): 185.7, 162.6, 137.0, 131.9, 119.0, 60.2; HR-MS (EI) $m / z[\mathrm{M}]^{+}$ calcd for $\mathrm{C}_{15} \mathrm{H}_{16} \mathrm{~N}_{2} \mathrm{O}_{2} \mathrm{~S} 288.0932$, found 288.0928.

1,3-Bis-(p-chlorophenyl)thiourea (13d) ${ }^{36}$ Colorless solid, mp $167-169{ }^{\circ} \mathrm{C}\left(172{ }^{\circ} \mathrm{C}\right): \delta_{\mathrm{H}}\left(400 \mathrm{MHz}, \mathrm{CDCl}_{3}\right): 7.92(\mathrm{~s}, 2 \mathrm{H}), 7.41-7.32$ $(\mathrm{m}, 8 \mathrm{H}) ; \delta_{\mathrm{C}}\left(100 \mathrm{MHz}, \mathrm{CDCl}_{3}\right): 180.2,135.6,132.3$, 129.5, 125.9; HR-MS (EI) $m / z[\mathrm{M}]^{+}$calcd for $\mathrm{C}_{13} \mathrm{H}_{10}{ }^{35} \mathrm{Cl}_{2} \mathrm{~N}_{2} \mathrm{~S}$ 295.9942, found 295.9940.

2-Phenylpent-4-enal (12a). ${ }^{17,18}$ Colorless oil, $\delta_{\mathrm{H}}(400 \mathrm{MHz}$, $\left.\mathrm{CDCl}_{3}\right) 9.69$ (d, 1H, J 1.6), 7.41-7.18 (m, 5H), 5.70 (m, 1H), 5.07$4.99(\mathrm{~m}, 2 \mathrm{H}), 3.61$ (t, 1H, J 7.2), 2.84 (q, 1H, J 6.8), 2.50 (q, 1H, J 6.8); $\delta_{\mathrm{C}}\left(100 \mathrm{MHz}, \mathrm{CDCl}_{3}\right) 201.8,136.6,135.7,129.0,128.8,127.6$, 117.1, 58.7, 33.9; HR-MS (EI) $m / z[\mathrm{M}]^{+}$calcd for $\mathrm{C}_{11} \mathrm{H}_{12} \mathrm{O}$ 160.0888 , found. 160.0885 .

2-p-Methoxyphenylpent-4-enal (12b). ${ }^{17}$ Pale yellow oil, $\delta_{\mathrm{H}}(400$ $\left.\mathrm{MHz} \mathrm{CDCl}_{3}\right) 9.62$ (d, 1H, J 1.6), 7.14 (m, 2H), 6.88 (m, 2H), 5.71 (m, 1H), $5.03(\mathrm{~m}, 2 \mathrm{H}), 3.78(\mathrm{~s}, 3 \mathrm{H}), 3.56(\mathrm{~m}, 1 \mathrm{H}), 2.85(\mathrm{~m}, 1 \mathrm{H})$, $2.46(\mathrm{~m}, 1 \mathrm{H}) ; \delta_{\mathrm{C}}\left(100 \mathrm{MHz}, \mathrm{CDCl}_{3}\right)$ 200.2, 159.1, 135.3, 129.9, $127.5,117.1,114.4,57.7,55.3$, 33.9; HR-MS (EI) $m / z[\mathrm{M}]^{+}$calcd for $\mathrm{C}_{12} \mathrm{H}_{14} \mathrm{O}_{2}$ 190.0994, found. 190.0988.

2-p-Chlorophenylpent-4-enal (12c). ${ }^{18}$ Colorless oil, $\delta_{\mathrm{H}}(400$ $\left.\mathrm{MHz}, \mathrm{CDCl}_{3}\right) 9.60$ (d, 1H, J1.6), 7.31-7.18 (m, 4H), $5.71(\mathrm{~m}, 1 \mathrm{H})$, $5.00(\mathrm{~m}, 2 \mathrm{H}), 3.62(\mathrm{~m}, 1 \mathrm{H}), 2.84(\mathrm{~m}, 1 \mathrm{H}), 2.49(\mathrm{~m}, 1 \mathrm{H}) ; \delta_{\mathrm{C}}(100$ $\mathrm{MHz}, \mathrm{CDCl}_{3}$ ) 200.1, 134.7, 134.5, 134.0, 130.8, 129.6, 118.1, 58.5, 34.5; HR-MS (EI) $\mathrm{m} / z[\mathrm{M}]^{+}$calcd for $\mathrm{C}_{11} \mathrm{H}_{11}{ }^{35} \mathrm{ClO} 194.0498$, found. 194.0491.

\section{Acknowledgements}

Support from the University of Kuwait, received through Research Grant no. SC 02/11, The Faculty of Graduate Studies for supporting the M. Sc. Student Nouf Al-Hamdan and facilities of GF-S (grant no. GS01/01, GS02/01, GS01/03, GS01/05) are gratefully acknowledged.

\section{References}

1 I. Ojima and F. Delaloge, Chem. Soc. Rev., 1997, 26, 377-386. 2 (a) M. D'hooghe, Y. Dejaegher and N. De Kimpe, Tetrahedron, 2008, 64, 4575-4584; (b) W. Van Brabandt and N. De Kimpe, J. Org. Chem., 2005, 70, 8717-8722. 
3 N. De Kimpe, Azetidines, Azetines and Azetes: Monocyclic Comprehensive Heterocyclic Chemistry II, ed. A. R. Katritzky, C. W. Rees and E. F. V. Scriven, Pergamon Press 1.18, 1996, pp. 507-572.

4 H. Staundinger, Liebigs Ann. Chem., 1907, 356, 51-123.

5 (a) Review: T. T. Tidwell, Eur. J. Org. Chem., 2006, 71, 563576; (b) L. Jiao, Q. F. Zhang, Y. Liang, S. W. Zhang and J. X. Xu, J. Org. Chem., 2006, 71, 815-818; (c) Y. Wang, Y. Liang, L. Jaoo, D.-M. Du and J. X. Xu, J. Org. Chem., 2006, 71, 6983-6990; (d) L. Jiao, Y. Liang, Q. Zhang, S. Zhang and J. X. Xu, Synthesis, 2006, 659-665; (e) L. Jiao, Y. Liang and J. X. Xu, J. Am. Chem. Soc., 2006, 128, 60606069; (f) Y. Liang, S. W. Zhang and J. X. Xu, J. Org. Chem., 2005, 70, 334-337; $(g)$ E. G. Lee, B. L. Hodoos, E. Bergan, C. Shih and G. C. Fu, J. Am. Chem. Soc., 2005, 127, 1158611587; (h) Y. A. Ibrahim, T. F. Al-Azemi, M. D. Abd ElHalim and E. John, J. Org. Chem., 2009, 74, 4305-4310; (i) Y. A. Ibrahim, T. F. Al-Azemi and M. D. Abd El-Halim, $J$. Org. Chem., 2010, 75, 4508-4513; (j) Y. A. Ibrahim, N. A. AlAwadi, T. F. Al-Azemi, S. Abraham and E. John, RSC Adv., 2013, 3, 6408-6416.

6 S. Lesniak, Pol. J. Chem., 1995, 69, 1490-1496.

7 S. Lesniak, Pol. J. Chem., 1995, 69, 1484-1489.

8 S. Lesniak, Pol. J. Chem., 1995, 69, 479.

9 R. Bartnik and S. Lesniak, Pol. J. Chem., 1994, 68(12), 26052611.

10 S. Lesniak, A. Chrostowska, D. Kuc, M. Maciejczyk, S. Khayar, R. B. Nazarski and L. Urbaniak, Tetrahedron, 2009, 65, 10581-10589.

11 M. Fischer, Chem. Ber., 1968, 101, 2669-2678.

12 L. A. Paquette, M. J. Wyvratt and G. R. Allen, J. Am. Chem. Soc., 1970, 92, 1763-1765.

13 Z. Yang and J. Xu, Tetrahedron Lett., 2012, 53, 786-789.

$14 \mathrm{H}$. Behbehani, M. R. Ibrahim and Y. A. Ibrahim, Tetrahedron Lett., 2002, 43, 6421-6426.

15 M. Suda, Chem. Lett., 1981, 7, 967-970.

16 R. E. Shade, A. M. Hyde, J. Olsen and C. A. Merlic, J. Am. Chem. Soc., 2010, 132, 1202-1203.

17 A. Joosten, È. Lambert, J. Vasse and J. Szymoniak, Org. Lett., 2010, 12, 5128-5131.

18 M. Zhang, Y. Hu and S. Zhang, Chem.-Eur. J., 2009, 15, 10732-10735.
19 Z. Guan, H. Lei, M. Chen, Z. Ren, Y. Bai and Y. Wang, Adv. Synth. Catal., 2012, 354, 489-496.

20 Z. Xiaohu, D. Liu, H. Guo, Y. Liu and W. Zhang, J. Am. Chem. Soc., 2011, 133, 19354-19357.

21 S. Mukherjee and B. List, J. Am. Chem. Soc., 2007, 129, 1133611337.

22 (a) P. Johannesson, G. Lindeberg, A. Johansson, G. V. Nikiforovich, A. Gogoll, B. Synnergren, M. LeGreves, F. Nyberg, A. Katlen and A. Hallberg, J. Med. Chem., 2002, 45, 1767-1777; (b) E. Marcantoni, M. Massaccesi and L. Sambri, J. Org. Chem., 2000, 65, 4553-4559; (c) E. O. Hormi and L. W. Hirvel, Tetrahedron Lett., 1993, 34, 6463-6466; (d) D. Gopal and Z. Rajagopalan, Tetrahedron Lett., 1987, 28, 5327-5330.

23 (a) W. Bao, Y. Liu and X. Lv, Synthesis, 2008, 1911-1917; (b) E. Mottia and M. Catellani, Adv. Synth. Catal., 2008, 350, 565-569; (c) S. B. Rodil, D. Peña and E. Guitián, Synlett, 2007, 8, 1308-1310.

24 H. Qi, Z. Yang and J. Xu, Synthesis, 2011, 723-730.

25 V. V. Govande, M. Arun, A. R. A. Deshmukh and B. M. Bhawal, Synth. Commun., 2000, 30, 4177-4182.

26 V. K. Ahluwalia, N. Mallika, R. P. Singh and V. D. Mehta, J. Indian Chem. Soc., 1989, 66, 200-201.

27 R. K. Goel, J. Pharm. Pharm. Sci., 2004, 7, 80-83.

28 Y. A. Ibrahim, N. A. Al-Awadi and K. Kaul, Tetrahedron, 2001, 57, 7377-7381.

29 Y. A. Ibrahim, K. Kaul and N. A. Al-Awadi, Tetrahedron, 2001, 57, 10171-10176.

30 Y. A. Ibrahim, N. A. Al-Awadi and M. R. Ibrahim, Tetrahedron, 2004, 60, 9121-9130.

31 W. Bao, Y. Liu and X. Lv, Synthesis, 2008, 1911-1917.

32 J. Wan, C. Wang and L. Yunyun, Appl. Organomet. Chem., 2012, 26, 445-447.

33 B. Alcaide, P. Almendros, C. Aragoncillo and N. R. Salgado, J. Org. Chem., 1999, 64, 9596-9604.

34 D. Ma, Q. Caia and X. Xie, Synlett, 2005, 11, 1767-1770.

35 B. Alcaide, J. Perez-Castells, C. Polanco and M. A. Sierra, J. Org. Chem., 1995, 60, 6012-6016.

36 F. Liang, J. Tan, C. Piao and Q. Liu, Synthesis, 2008, 22, 35793584.

37 M. A. Pasha and V. P. Jayashankara, Synth. Commun., 2006, 36, 1787-1793. 